\title{
RIESGO Y RENDIMIENTO DE LOS FONDOS DE PENSIONES EN MÉXICO: ANÁLISIS DE LA SIEFORE BÁSICA 3
}

\section{Risk and Return of Pension Funds in Mexico: Analysis of the Siefore Basica 3}

\author{
Raúl Arturo Cornejo López ${ }^{1}(\dagger)$ \\ Israel Bermúdez Pacheco ${ }^{2}$
}

\section{RESUMEN}

El objetivo del presente artículo es analizar la relación riesgo-rendimiento de 10 Sociedades de inversión especializadas en fondos para el retiro (Siefores) pertenecientes a la Siefore Básica 3 (SB3) desde su creación en marzo de 2008 hasta junio de 2019. Lo anterior, con el propósito de comprobar si el postulado financiero establecido por Harry Markowitz de a mayor riesgo, mayor rendimiento se cumpliría en ese lapso. Para estimar el riesgo de mercado, al igual que los rendimientos, se utilizó la metodología econométrica de los modelos ARIMA-GARCH, cuyas pruebas de raíces unitarias mostraron evidentes rompimientos estructurales que fueron incluidos en los modelos presentados. Los resultados confirman que los fondos de pensiones que expusieron sus inversiones a mayores riesgos no generaron mayores rendimientos, lo que podría evidenciar una operación deficiente en el proceso de inversión por parte de las Siefores que afectaría el monto de la pensión del trabajador en el largo plazo. Finalmente, los rendimientos estimados de los 10 fondos se compararon contra el Indicador de Rendimiento Neto de la CONSAR encontrándose altas coincidencias en dicha comparativa.

\footnotetext{
${ }^{1}$ Doctor en Ciencias de la Administración; Profesor-Investigador en la UAM-Iztapalapa, Coordinación de Administración, Área Planeación Estratégica de las Empresas, y de la FCA de la UNAM; Miembro del SNI. Correo electrónico: racornejo@hotmail.com

2 Maestro en Finanzas Corporativas con especialidad en Economía Monetaria y Financiera, FCAUNAM. Correo electrónico: israbermudex@gmail.com
} 
Palabras clave: Siefores, Riesgo, Rendimiento, Modelos ARIMA-GARCH, Rompimiento Estructural.

Clasificación JEL: C58, G11, G23

\section{ABSTRACT}

The fundamental purpose of this paper is to analyze the risk-return relation of 10 Mexican Pension Funds Societies (Siefores) belonging to the Basic Siefore 3 (SB3) from its creation in March 2008 up to June 2019. The foregoing, with the purpose of verifying if the investment postulate by Harry Markowitz was fulfilled. The postulate indicates that the greater the risk, the greater the return obtained. To estimate the market risk as well as the returns, the econometric methodology of the ARIMA-GARCH models was used. The unit root tests showed structural changes that were included in the models. The results confirm that the pension funds that exposed their investments to higher risks did not receive higher returns, showing a bad operation of the investment process by the Siefores which would affect the amount of the worker's pension in the long term. Finally, the estimated returns of the 10 funds were compared against the CONSAR Net Performance Indicator and in all cases, high coincidences were found in the comparison.

Keywords: Siefores, Risk, Returns, ARIMA-CARCH Models, Structural Break. JEL Classification: C58, G11, G23

\section{Introducción}

A partir de la reforma implementada al sistema de pensiones en julio de 1997 , los fondos de pensiones cobraron relevancia en la economía mexicana. Dicha reforma estableció un nuevo sistema de contribuciones definidas que reemplazó al sistema de pensiones de beneficios definidos vigente desde 1943 (Santillán, Martínez y López, 2016). La principal característica del nuevo sistema fue su administración por empresas privadas a través de cuentas individuales que concentran las aportaciones tripartitas de trabajadores, empleadores y gobierno (Martínezy Venegas, 2014). Estas cuentas son manejadas por Administradoras de Fondos para el Retiro (Afores o Administradoras, en adelante) que invierten los recursos de los trabajadores a través de Sociedades de Inversión Especializadas en Fondos para el Retiro (Siefores) o Sociedades, las cuales son reguladas por la Comisión Nacional del Sistema de Ahorro para el Retiro (CONSAR). 
Al primer semestre de 2019, se encuentran operando cinco Siefores básicas que son asignadas de acuerdo con el rango de edad del trabajador: la Siefore Básica o (SBO) y la Siefore Básica 1 (SB1) para personas de 60 años y mayores; la Siefore Básica 2 (SB2) para personas entre 46y 59 años; la Siefore Básica 3 (SB3) para personas entre 37 y 45 años y la Siefore Básica 4 (SB4) para personas de 36 años y menores ${ }^{3}$. Esta familia de Siefores se creó para buscar que el Sistema de Ahorro para el Retiro (SAR) fuera financieramente viable invirtiendo los ahorros de en instrumentos financieros con el objetivo de generar rendimientos que al final incrementen el monto de la pensión del trabajador. Los recursos acumulados en el SAR han aumentado año con año alcanzando aproximadamente 3.1 billones de pesos (bdp) a diciembre de 2017 (véase Gráfica 1). Dicha cifra representa el $14.7 \%$ del Producto Interno Bruto (PIB) de México. Sin embargo, el punto relevante es el comportamiento que tuvieron los rendimientos generados por las inversiones en los 20 años de operación y que equivalen aproximadamente a $1.4 \mathrm{bdp}$. En términos porcentuales, los rendimientos representaron el $45 \%$ del total de los recursos, de ahí su importancia como objeto de estudio.

\section{GRÁFICA 1. APORTACIONES Y RENDIMIENTOS EN EL SAR}

ACTIVOS NETOS EN MILLONES DE PESOS, 1997-2017

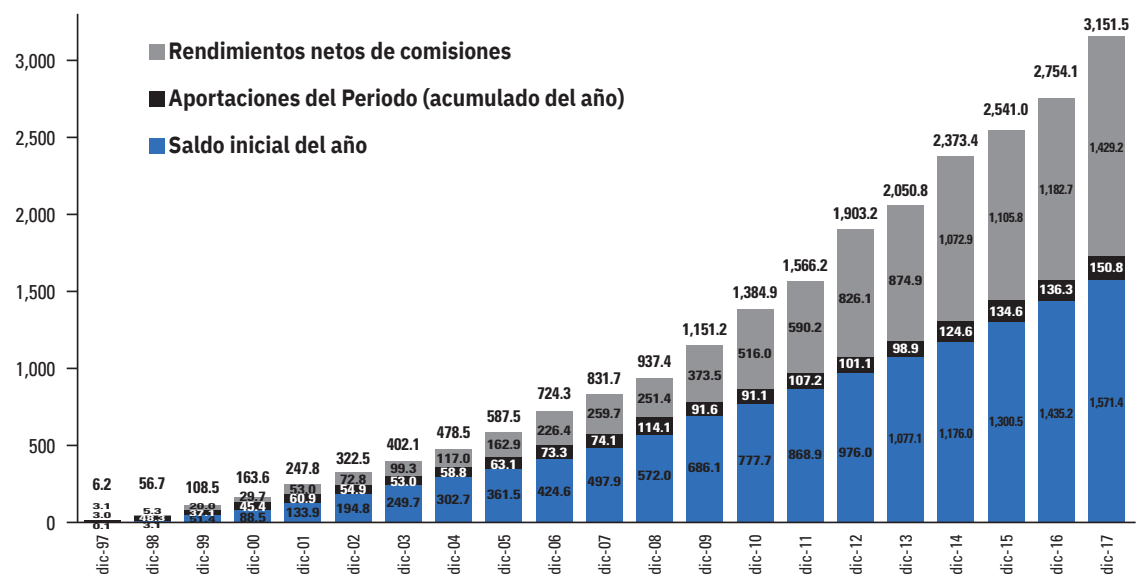

Fuente: CONSAR (2018)

\footnotetext{
${ }^{3}$ A partir de diciembre de 2019 se pasó de cinco Siefores básicas a 10, que cambian cada cinco años: de la Básica Inicial, para los trabajadores que tienen menos de 25 años, a la Básica de Pensiones, para los que tienen 65 años o más (nota del editor).
} 
De acuerdo con la Comisión Nacional del Sistema de horro para el Retiro (CONSAR, 2018), los rendimientos históricos obtenidos de julio de 1997 a junio de 2018 han sido competitivos ya que el rendimiento nominal anual promedio generado por el SAR fue aproximadamente $11.33 \%$ y en términos reales se ubicó en $5.43 \%$. Sin embargo, de 2003 a 2016 los rendimientos históricos nominales mostraron una evidente tendencia a la baja ya que pasaron del $17.9 \%$ a un $10.6 \%$ (véase Gráfica 2), una caída cercana al $40 \%$. Respecto a los rendimientos reales (que restan el efecto de la inflación) decrecieron aproximadamente $42 \%$ pues en 13 años pasaron del $8.1 \%$ a un $4.7 \%$. Otro punto para destacar es que los rendimientos reales se estancaron entre 2008 y 2016 , observando en promedio un crecimiento del $5.4 \%$ en dicho periodo.

GRÁFICA 2. RENDIMIENTOS HISTÓRICOS DEL SAR: 2003-2016

Precios de Bolsa EN PORCENTAJE

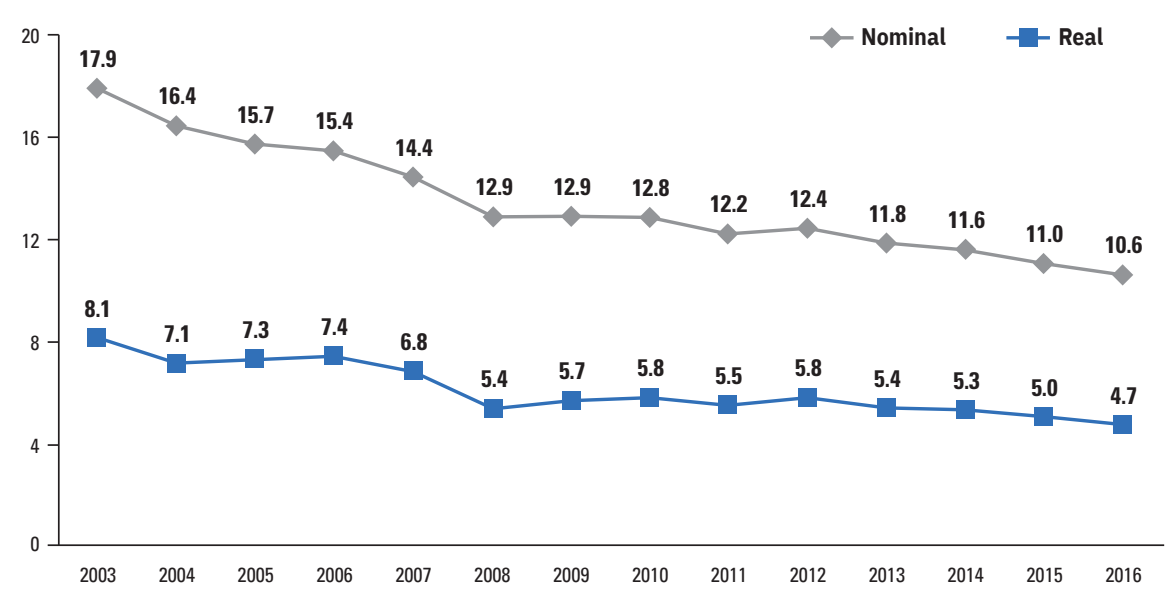

Fuente: CONSAR (2017)

La problemática detectada de los rendimientos decrecientes y estancados se considera preocupante para el funcionamiento óptimo del SAR que, como consecuencia, podría afectar el ahorro del trabajador, ya que como se mencionó, los rendimientos generados por las Siefores representan una parte significativa de su ahorro. Además, se evidencia la importancia de una probable e inadecuada gestión de los fondos de pensiones, lo que denota la necesidad de un mayor co- 
nocimiento sobre la generación de rendimientos por parte de las Sociedades y del riesgo al que exponen las inversiones. Los portafolios de las Siefores están diversificados en inversiones de renta fija y variable expuestas al riesgo de mercado debido a la volatilidad de los mercados financieros nacionales e internacionales. De acuerdo al régimen de inversión de las Siefores, si el riesgo al que se invierten los ahorros es mayor, los rendimientos obtenidos deberían ser los más altos, de no ser así, se estarían arriesgando los ahorros.

Bajo el contexto anterior, es importante analizar los rendimientos generados por las Sociedades para conocer si efectivamente el riesgo de mercado ha sido compensado por los rendimientos obtenidos. Así, la pregunta de investigación que se planteó es este trabajo es la siguiente: ¿Cuál fue el rendimiento de las Siefores que conformaron la SB3, en función del riesgo de mercado entre 2008 y 2019? Por su parte, la hipótesis que da respuesta a la pregunta de investigación fue la siguiente: La relación riesgo-rendimiento no se cumple para las Siefores que conformaron la SB3 porque las inversiones con mayor riesgo, no generaron mayores rendimientos. Por lo tanto, el objetivo de la investigación fue analizar la relación riesgo-rendimiento de diez Siefores que conformaron la SB3, calculando el rendimiento y el riesgo de mercado de sus inversiones de 2008 a 2019.

La estructura del trabajo es la siguiente: la siguiente sección presenta los estudios previos relacionados con el tema de investigación, un breve panorama general del SAR y la creación y evolución de las Siefores en México. En la tercera sección se presenta el marco teórico. La metodología econométrica empleada es explicada en la cuarta sección. La quinta sección presenta el análisis empírico aplicado a la SB3. Por último, las conclusiones forman parte final del documento. Es importante señalar que los resultados obtenidos en esta investigación no pretenden funcionar como una guía para la selección de una Siefore óptima para el ahorrador, tampoco buscan generar ninguna tendencia económica ni política al respecto.

\section{Revisión de la literatura}

En la investigación de Banda y Cómez (2009) se analizaron los portafolios de inversión de la SB2 de 2000 a 2009 utilizando los índices de Sharpe, Treynor y Jensen con el fin de determinar cuál Siefore obtuvo un desempeño óptimo. Los resultados mostraron una escasa relación entre los rendimientos del mercado y los rendimientos de los fondos. Sólo una Sociedad obtuvo un rendimiento por 
encima del rendimiento del activo libre de riesgo que fueron Certificados de la tesorería de la federación (Cetes) a 28 días y ninguna sobrepasó el rendimiento del mercado medido a través del Índice de Precios y Cotizaciones (IPC). La investigación señaló que no fue posible concluir cual Sociedad fue la mejor ya que el ajuste del rendimiento con respecto al riesgo difirió en los tres índices utilizados. Por último, se indicó que el estudio puede ser enriquecido mediante el análisis de las Siefores Básicas 3, 4 y 5.

El artículo de Banda, Conzález y Cómez (2014) presentó una aplicación de la Teoría de la Cartera para las Siefores con el fin de que los trabajadores conformen un portafolio personalizado con una combinación óptima de recursos a invertir. El periodo de estudio comprendió de marzo de 2009 a mayo de 2012 para las cinco Siefores Básicas y los resultados mostraron la existencia de una frontera eficiente derivada de la combinación de los diferentes activos. Sin embargo, la legislación actual del SAR no permite a los ahorradores combinar activos para conformar portafolios; por lo tanto, se dificulta emplear en la práctica la metodología propuesta por dichos autores.

En el estudio de Martínez y Venegas (2014) se construyó un índice con los precios de cierre diarios (de 1997 a 2010 para la SB1 y de 2004 a 2010 para la SB2) que fue modelado con procesos ARIMA-CARCH y varias de sus extensiones con el fin de examinar el comportamiento de los rendimientos y la volatilidad. Uno de los resultados relevantes fue que los rendimientos obtenidos no fueron suficientes para compensar el riesgo adicional asumido por los fondos de pensiones que incluyeron componentes de renta variable.

Herrera (2015) analizó la relación riesgo-rendimiento de la SB4 (de diciembre de 2012 a diciembre de 2014). El estudio consistió en la construcción de un portafolio con Cetes y opciones cuyo activo subyacente fue la acción de -América Móvil-, donde al total de la inversión se le restó la tasa esperada de Cetes y de esta diferencia se invirtió la mitad en calls y la otra en puts. Sin embargo, Cómez (2016) señaló que la estrategia propuesta anteriormente no fue la óptima. El artículo de Santillán, Martínez y López (2016) presentó un estudio econométrico para analizar los rendimientos y la volatilidad de las cinco Siefore Básicas (SB1 de 1997 a 2012, SB2 de 2004 a 2012 y las SB3, SB4 y SB5 de 2008 a 2012) con el objetivo de determinar la presencia de efectos de memoria de largo plazo. Se encontró evidencia de que tanto en los rendimientos como en la volatilidad están presentes efectos de memoria de largo plazo; es decir, las variables están auto-correlacionadas con sus propios valores en periodos del pasado. Finalmente, en el trabajo 
de Romero (2016) se analizó el riesgo y rendimiento de los portafolios de la SB4 y SB5 (entre 2008 y 2012) utilizando índices de Sharpe y Treynor se llegó a la conclusión que el rendimiento generado estuvo en proporción al riesgo asumido; no obstante, el portafolio de las Siefores se comparó contra el portafolio del IPC que no fue completamente equiparable, pero se utilizó ya que no se encontró uno que midiera los fondos de pensiones.

De las investigaciones presentadas, sólo dos analizaron la SB3. Considerando lo anterior, esta investigación propuso enriquecer el estudio de la SB3 analizándola desde su creación en 2008 hasta el primer semestre de 2019. De este modo, se consideraron los efectos de memoria de largo plazo en los rendimientos y la aportación de este trabajo consistió en estimar el riesgo de mercado para conocer si en verdad fue compensando por los rendimientos obtenidos durante los 11 años que ha operado dicho fondo. En línea con lo anterior, la única Siefore que ha operado de manera continua desde su creación hasta 2019 es la SB3, esto representa una justificación para su estudio, ya que las demás Sociedades fueron absorbidas, divididas o fusionadas provocando una combinación de recursos y rendimientos que dificultan un análisis cuantitativo más acertado.

\subsection{Panorama general del SAR en México}

Bonifant y Cómez (2010) señalan que el Sistema de Ahorro para el Retiro (SAR) en México tiene sus orígenes en la Constitución de 1917, ya que la fracción XXIX del Artículo 123 establece que es indispensable que exista una Ley del Seguro Social que comprenda seguros de vejez y protección a los trabajadores. Lo anterior se llevó a cabo hasta 1943 cuando se creó la Ley del Seguro Social. Posteriormente, en 1995 se aprobó una reforma a dicha Ley que estableció dos aspectos fundamentales para las pensiones. El primero de ellos consistió en la transición del Sistema de Beneficio Definido (SBD), al nuevo Sistema de Contribución Definida (SCD) también conocido como Ceneración Afore, que entró formalmente en operaciones en 1997 e incluye a los trabajadores que empezaron a cotizar al Instituto Mexicano del Seguro Social a partir del 1 de julio de 1997y a los trabajadores cotizantes al Instituto de Seguridad y Servicios Sociales de los Trabajadores del Estado a partir del 1 de abril de 2007. El segundo aspecto importante fue el establecimiento del SAR para brindar servicios a los trabajadores que cotizan en ambos Institutos y a los trabajadores independientes quienes también puede tener acceso a una Afore. 
En el SBD los trabajadores activos contribuían con sus ingresos para crear una reserva que pagaba las pensiones de las personas retiradas; sin embargo, factores como el incremento de la esperanza de vida, el aumento de trabajadores pensionados, la disminución de trabajadores activos y la canalización de los recursos de las pensiones hacia los servicios de salud por parte del gobierno, provocaron que las reservas del fondo se agotaran. En respuesta a esta problemática se transitó hacia el SCD para buscar que el esquema de pensiones fuera financieramente viable invirtiendo los ahorros de los trabajadores para generar rendimientos. Continuando con Bonifant y Gómez (2010), señalan que el SCD consiste en que cada trabajador disponga de una cuenta individual en la que se depositen las aportaciones tripartitas realizadas por el patrón, el trabajador y el gobierno por concepto de ahorro para el retiro. En el SCD, las Afore son sociedades anónimas de capital variable, que utilizan en su denominación o a continuación de ésta, la expresión "Administradora de Fondos para el Retiro" o su abreviatura "Afore". El proceso de inversión de las Afores parte del principio de responsabilidad fiduciaria donde las administradoras deben atender en todo momento al interés de los trabajadores. Actualmente operan en México diez Afores: Azteca, Citibanamex, Coppel, Inbursa, Invercap, PensionISSSTE Principal, ProfuturoCNP, Sura y XXI Banorte, las cuales han invertido a septiembre de 2018: 50.18\% de sus recursos en valores gubernamentales, $18.95 \%$ en deuda privada nacional y $14.09 \%$ en renta variable internacional (ver cuadro 1 ).

CUADRO 1. INVERSIONES REALIZADAS POR LAS SIEFORES

AL CIERRE DE SEPTIEMBRE DE 2018 EN PORCENTAJE.

\begin{tabular}{lc}
\hline Inversiones & $\%$ \\
\hline Renta variable nacional & 7.47 \\
\hline Renta variable internacional & 14.09 \\
\hline Mercancías & 0.3 \\
\hline Deuda privada nacional & 18.95 \\
\hline Instrumentos Estructurados & 5.49 \\
\hline FIBRAS & 2.86 \\
\hline Deuda internacional & 0.66 \\
\hline Valores gubernamentales & 50.18 \\
\hline Total & $\mathbf{1 0 0}$
\end{tabular}

Fuente: CONSAR (2018a) 
Las sociedades de inversión, administradas y operadas por las Afores deberán ser sociedades anónimas de capital variable y utilizar en su denominación, o a continuación de ésta, la expresión "Sociedad de Inversión Especializada de Fondos para el Retiro" o su abreviatura "Siefore" y tienen por objeto invertir las aportaciones destinadas a fondos de previsión social, las aportaciones voluntarias y complementarias de retiro que reciban de los trabajadores y patrones. Las Sociedades deberán contar con un comité de inversión para determinar la política y estrategia de inversión y la composición de los activos y contar también con un comité de riesgos para administrar los riesgos al que se encuentren expuestas y vigilar que la realización de sus operaciones se ajuste a los límites, políticas y procedimientos para la administración de riesgos. Por último, el ordenamiento jurídico aplicable al funcionamiento del SAR es la Ley de los Sistemas de Ahorro para el Retiro (LSAR) de 1996 y el regulador es la CONSAR o Comisión, creada en julio de 1994 como un órgano administrativo desconcentrado de la Secretaría de Hacienda y Crédito Público (SHCP), por eso se establece que existe una alta seguridad del ahorro.

\subsection{Creación y evolución de las Siefores: 1997-2019}

Durante el desarrollo de la investigación, se detectaron seis momentos importantes respecto a la creación y evolución de las Sociedades. El primero de ellos fue en 1997 cuando se crearon las primeras dos Siefores asignadas de acuerdo al rango de edad de los trabajadores: la SB1 para el grupo de 56 años en adelante y la SB2 de 55 años y menores. El segundo momento fue en 2008 cuando se crearon tres Sociedades: la SB3 para trabajadores de 37 y 45 años; la SB4 de 27 y 36 años y la SB5 para ahorradores jóvenes de 26 años y menores. La creación de los tres fondos fue con el objetivo de ofrecer al trabajador acceso a mayores rendimientos y riesgos de acuerdo con su rango de edad; por lo tanto, se esperaría que en los próximos años los rendimientos se incrementaran (o por lo menos se mantuvieran estables), pero como se mencionó, a partir de 2008 empezaron a descender y se estancaron. El tercer momento ocurrió en octubre de 2012, cuando la SB4 fue absorbida por la SB5 debido a la baja cotización de ahorradores de 26 años y menores que limitó el aprovechamiento del régimen de inversión. El cuarto momento fue en diciembre de 2015 cuando se creó la Siefore Básica de Pensiones o SBo para blindar de la volatilidad de los mercados financieros los recursos de trabajadores de 60 años 0 más que están próximos al retiro. Así, a enero de 2019, el SAR contaba con cinco 
Siefore Básicas que funcionaban bajo un sistema de multifondos que atendían distintos grupos de cotizantes. Las reglas de inversión de cada fondo iban cambiando conforme los ahorradores se encontraban más cerca del retiro: entre más joven, mayor exposición a instrumentos de inversión de largo plazo con mayor rendimiento y un mayor riesgo (véase Figura 1), mientras los trabajadores más cercanos al retiro tenían su ahorro invertido en modalidades de corto plazo con un menor nivel de riesgo y por lo general, un menor rendimiento.

Figura 1. Ciclo de Vida de los trabajadores y familia de Siefores a 2018

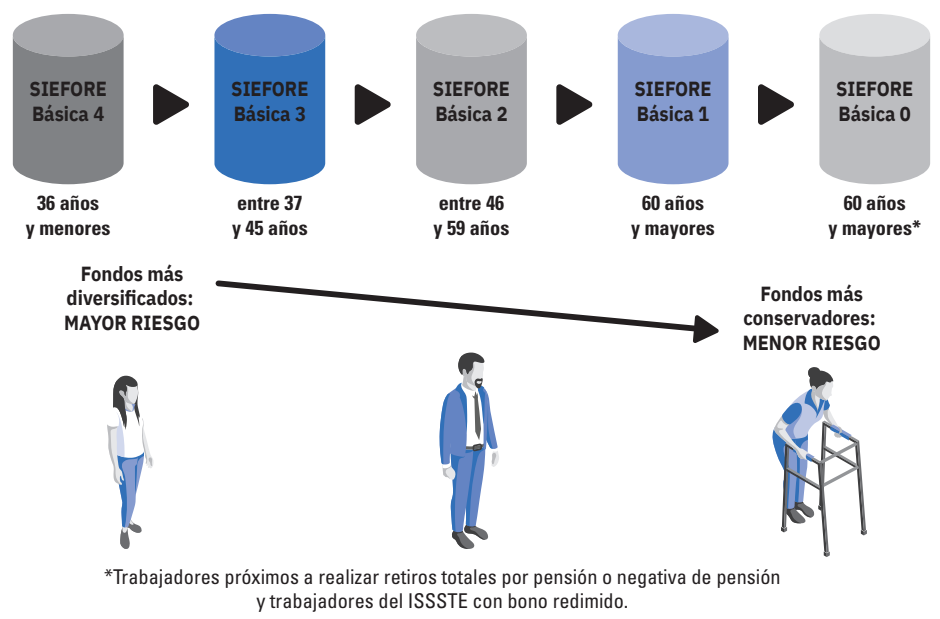

* Trabajadores próximos a realizar retiros totales por pensión o negativa de pensión y trabajadores del ISSSTE con bono redimido.

Fuente: e-SAR (2018).

El quinto momento fue en enero de 2017, cuando la CONSAR estableció el esquema de Libre Selección de Siefore (LSS). Antes de 2017, las Siefores eran asignadas de acuerdo con el rango de edad del trabajador; sin embargo, a partir de 2017 la Comisión instauró la LSS como una herramienta a través de la cual el ahorrador podrá transferir el ahorro de su Siefore a otra sin importar su edad. El cambio de Sociedad se podrá realizar cada 3 años de forma gratuita y para los ahorradores que no ejerzan esta nueva opción, su proceso de selección de Siefore seguirá siendo de acuerdo con su rango de edad. La CONSAR (2017) creó la LSS con el objetivo de otorgar mayor poder de decisión a los trabajadores respecto 
a su ahorro y brindar la posibilidad de alcanzar un mayor rendimiento a cambio de un mayor nivel de riesgo. Por ejemplo, se puede decidir que los recursos estén invertidos en la SB4 (que invierte en proyectos productivos de larga maduración) a pesar de que por edad le corresponda al trabajador la SB3 (e-SAR, 2018). No obstante, con el mecanismo de LSS, no se sabe si le conviene al trabajador cambiarse de Sociedad y asumir más riesgo, pues esta decisión depende sólo si los rendimientos que espera obtener, compensarán el mayor riesgo.

Por último, el sexto momento ocurrió en mayo de 2019 cuando la junta de Cobierno de la CONSAR reformó la LSAR para transitar del esquema de multifondos de las Siefores a un esquema de fondos generacionales, el cual propone lograr una -transición suave- en las inversiones realizadas por las Siefores, para reemplazar la -desinversión abrupta- que se realizaba bajo el esquema de multifondos asignados por rangos de edades (véase Gráficas 3 y 4). De acuerdo con la CONSAR (2019), México es el primer país en Latinoamérica en adoptar el esquema de fondos generacionales que tiene entre sus objetivos: fomentar el diseño de una estrategia de inversión de largo plazo por parte de las Administradoras que se adecúe a la evolución del perfil de riesgo-rendimiento de los trabajadores durante su vida laboral; ofrecer más alternativas de inversión en búsqueda de mayores rendimientos; mejores pensiones para los trabajadores (mayor tasa de reemplazo); otorgar mejores herramientas de defensa ante ciclos de volatilidad en los mercados y realizar precisiones que otorguen certeza jurídica a los regulados.

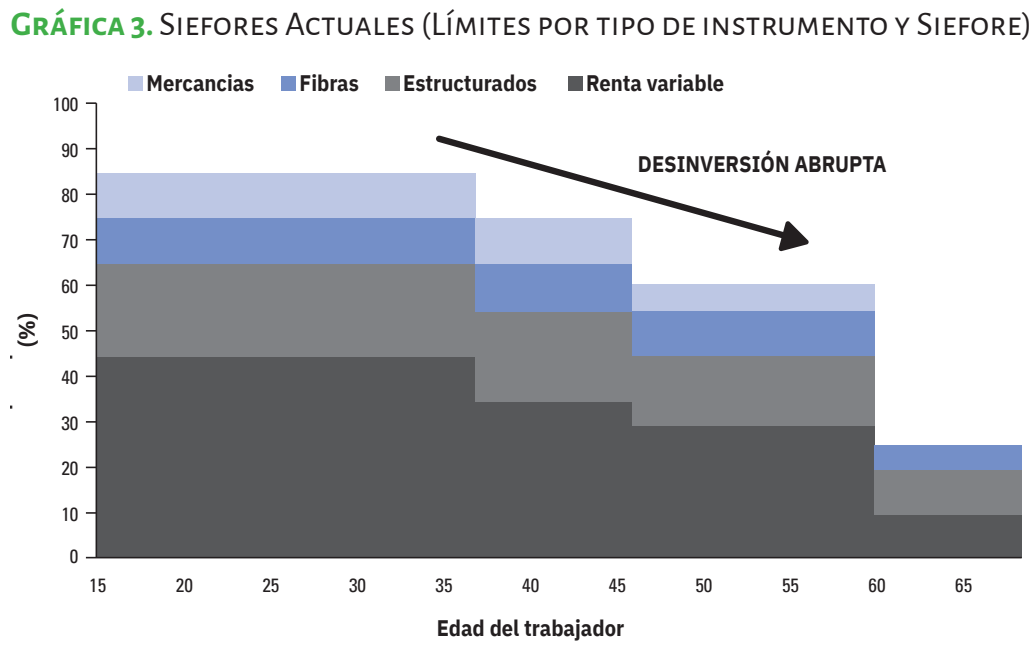

Fuente: CONSAR (2019) 
Gráfica 4. Siefores Generacionales (Límites Por tipo de INSTRUMENTO y SiEFore)

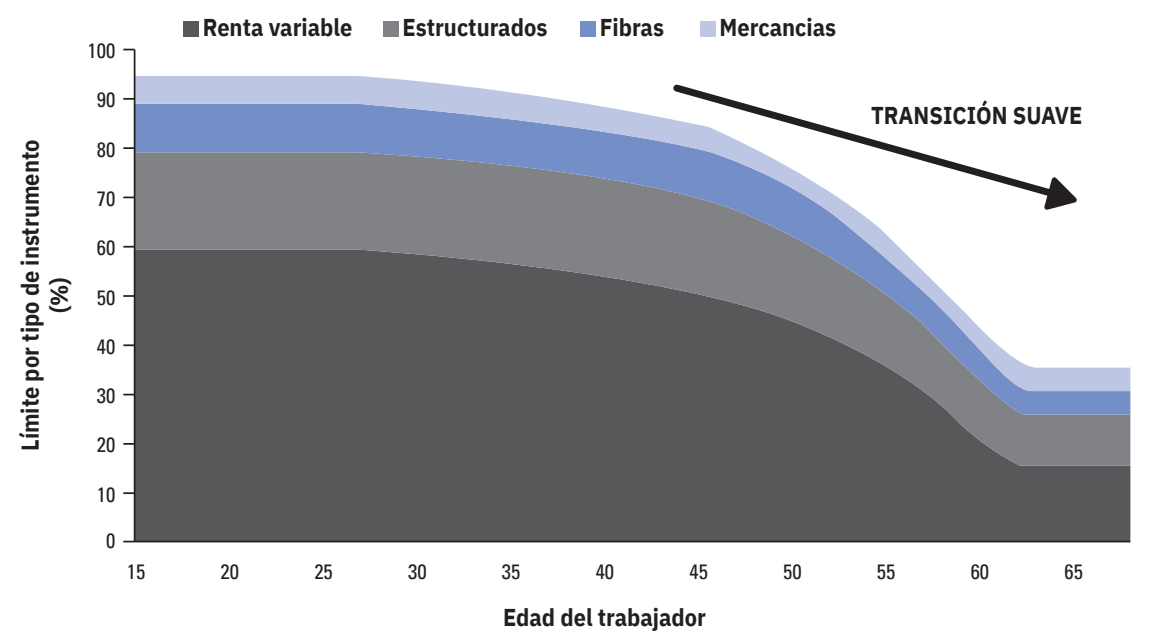

Fuente: CONSAR (2019)

\section{Marco teórico}

El rendimiento de un activo financiero se define como el cambio de valor que registra en un período con respecto a su valor inicial (UNAM, 2015), generalmente es expresado en términos de porcentaje sobre lo invertido y representa la ganancia o pérdida resultante de una inversión (Véliz, Cervantes y Carmona 2012). En el caso del sistema de pensiones, los rendimientos son las ganancias o intereses generados por la inversión de los ahorros. Por su parte, el riesgo financiero se relaciona con las pérdidas potenciales que puede sufrir un portafolio de inversión, debido a la volatilidad de los flujos financieros no esperados. El riesgo financiero se define como "la pérdida potencial en el valor de los activos financieros debido a movimientos adversos en los factores de riesgo que determinan su precio, por ejemplo: las tasas de interés o el tipo de cambio" (Banxico, 2005, p.5).

Ahora bien, para construir un portafolio de inversión es indispensable tomar en cuenta la relación riesgo-rendimiento ya que de nada vale obtener un determinado rendimiento sin tener presente los riesgos asociados (UNAM, 2015). Cabe señalar que dicha relación se fundamenta en una de las teorías aplicables a la gestión de riesgos de activos financieros: la Teoría de la Cartera (Portfolio Theory, 
por sus siglas en inglés). A partir del modelo planteado por John Burr Williams (1938) en su libro Theory of Investment Value, Markowitz (1952) y Tobin (1958) desarrollaron los conceptos básicos de la Teoría de la Cartera (Banda, Conzález y Gómez, 2014). Asociando el riesgo a la varianza del valor de una cartera y basándose en la búsqueda de la evasión del riesgo, derivaron la Teoría de la Cartera (TDC, en adelante) que estudia como maximizar la rentabilidad y minimizar los riesgos mediante la composición óptima de una cartera de valores. En línea con lo anterior, en 1959 Markowitz en su obra Portafolio Selection: Efficient diversification of investments planteó la importancia de la diversificación de los portafolios para disminuir el riesgo y aumentar el rendimiento de la cartera (Banda, Conzález y Gómez, 2014). Algunas hipótesis sobre el comportamiento y el método racional de elección del inversor de la TDC señaladas por Blanco, Ferrando y Martínez (2015) son las siguientes:

- Todos los individuos se comportan racionalmente y son maximizadores de su función de utilidad esperada

- La función de utilidad esperada del inversor depende únicamente del rendimiento esperado (como medida de la rentabilidad) y la varianza o desviación típica (como medida del riesgo)

- Los inversores tienen aversión al riesgo, por lo que, para una cartera de valores con un mismo rendimiento esperado, preferirán la cartera con menor varianza

En general, la TDC ha señalado que considerar los atributos de riesgo y rendimiento pueden ser suficientes para tomar muchas decisiones financieras, el problema estriba en la adecuada combinación de dichas variables. (Ferruz y Sarto, 2002). El propio Markowitz (1952) señaló acertadamente la necesidad de considerar la función de utilidad de los inversores para determinar la combinación óptima entre riesgo y rendimiento. En este sentido analizó por primera vez estos dos atributos y graficó el riesgo en abscisas y el rendimiento en ordenadas y encontró que existía una relación directamente proporcional, de manera que, para aceptar niveles mayores de riesgo, cualquier inversor exigirá un rendimiento esperado superior; es decir, el postulado financiero de las inversiones que estable lo siguiente: a mayor riesgo mayor rendimiento y viceversa. Un ejemplo de lo anterior se muestra en la Gráfica 5 donde una inversión en Cetes o bonos gubernamentales (considerados como instrumentos de bajo riesgo) obtendrá rendimientos 
esperados menores en comparación con una inversión en derivados financieros los cuales se consideran como instrumentos de mayor riesgo.

\section{Gráfica 5. RELACIÓN (EX ANTE) RIESGO-RENDIMIETO ESPERADO PARA ACTIVOS FINANCIEROS.}

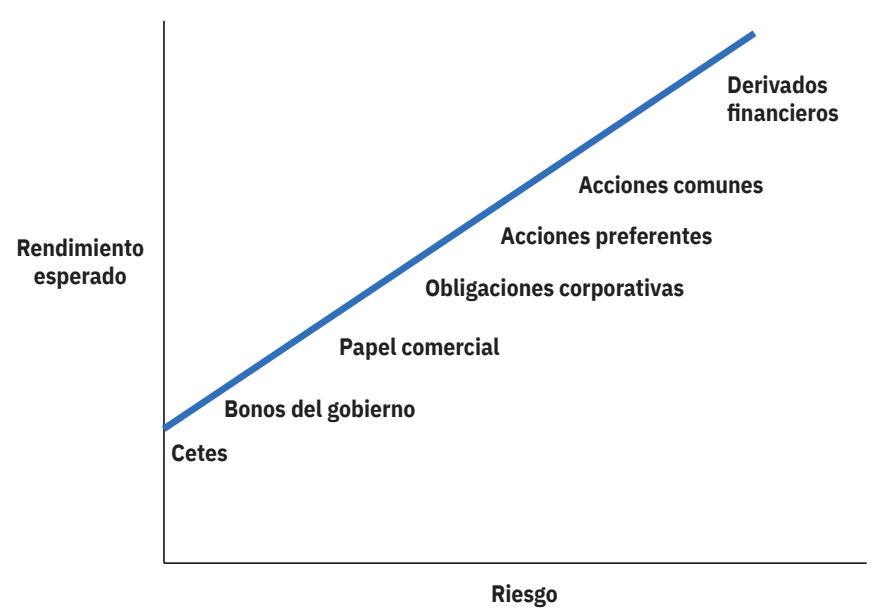

Fuente: Administración y Economía (2013)

\section{Metodología ARIMA-GARCH}

En series de tiempo financieras, se busca trabajar con series estacionarias que, por lo general, resultan de la diferencia de los logaritmos que justamente coincide con el cálculo de los rendimientos de un activo financiero. Primero se obtienen logaritmos para estabilizar la varianza ya que el logaritmo permite reducir el nivel de volatilidad sin cambiar la estructura del comportamiento de la serie. Posteriormente, para obtener los rendimientos se calcula la diferencia de la serie logarítmica para estabilizar el término de la media. De esta manera, se puede realizar una estimación de los rendimientos más ad hoc en el tratamiento de series financieras. A continuación, se presenta la metodología para el cálculo de rendimientos de activos financieros propuesta por Jorion (2004). Para calcular un horizonte de largo plazo, en la práctica se utiliza la tasa de rendimiento geométrico, la cual se define en términos de logaritmos del cociente de precios: 
Donde:

$$
r t=\ln \left[\frac{P t+D t}{P t-1}\right]
$$

$r t$ es el rendimiento del activo financiero $i$ al tiempo $t$, In es el logaritmo del cociente de precios, Pt es el precio de la cartera del activo financiero al tiempo $t, y$ $P t-1$ es el precio de la cartera del activo financiero al tiempo $t-1$

Una vez determinados los rendimientos continuos mensuales (por ejemplo, para un periodo de 5 años) se busca obtener el rendimiento promedio anualizado. Una buena aproximación para dicha estimación es la propuesta por Martínez y Venegas (2014):

Donde:

$$
r_{a}=\left[\frac{\sum \ln p_{f}-\ln p_{i}}{n}\right] * t p
$$

$r_{\mathrm{a}}$ es el rendimiento promedio anualizado del periodo, $\ln p_{f}$ el logaritmo en la última fecha del periodo, $\ln p_{i}$ el logaritmo en la fecha de inicio del periodo, $n$ el número de observaciones y tp representa el número de días promedio anual en el periodo estudiado

Ahora bien, el modelo Autorregresivo Integrado de Promedios Móviles (ARIMA o Autoregressive Integrated Moving Average, por sus siglas en inglés) se basa en el supuesto de que las series de tiempo consideradas son débilmente estacionarias; es decir, que su media y varianza son constantes y su covarianza es invariante en el tiempo. Si una serie de tiempo es integrada de orden 1 [si es I(1)] sus primeras diferencias son I(o) o estacionarias; es decir, es una serie de tiempo autorregresiva integrada de promedios móviles, donde $\mathrm{p}$ denota el número de términos autorregresivos, d el número de veces que la serie debe diferenciarse para hacerse estacionaria y q el número de términos de promedios móviles. La razón para suponer estacionariedad es explicada por Pokorny (1987) en Gujarati y Porter (2010) de la siguiente manera: "la sencilla razón para requerir datos estacionarios es que todo modelo que se infiera a partir de estos datos pueda interpretarse como estacionario o estable en sí mismo, y proporcione, por consiguiente, una 
base válida para pronosticar" (Gujarati y Porter, 2010, p.777). En esta investigación no se pretende desarrollar un pronóstico de los rendimientos bajo la premisa de que rendimientos pasados no garantizan rendimientos futuros; sin embargo, para el cálculo de los rendimientos financieros se utilizó un Modelo ARIMA de la forma $(p, 1, q)$, donde la serie fue diferenciada una vez para hacerla estacionaria. Por su parte, el modelo con Heterocedasticidad Condicional Autorregresiva, (ARCH o Autoregressive Conditional Heteroskedasticity, por sus siglas en inglés) fue desarrollado por Robert F. Engle en 1982. El éxito de la familia de modelos ARCH se puede atribuir a las aplicaciones que tienen en finanzas ya que pueden aplicarse a problemas estadísticos y adquieren un valor especial cuando se aplican a series temporales financieras. Lo anterior se debe en parte a la importancia de la relación riesgo-rendimiento en los mercados financieros y a tres características presentes en los rendimientos (Engle y Patton, 2001):

1. Agrupamiento o clúster de volatilidad: Existen lapsos donde los precios muestran amplias variaciones durante prolongados periodos y luego se dan intervalos de una calma relativa.

2. Exceso de curtosis: Los rendimientos tienen sorprendentemente, una gran cantidad de valores extremos o colas gordas.

3. Impredecibilidad: Los rendimientos son prácticamente impredecibles.

En los modelos ARCH, la heteroscedasticidad, o varianza desigual, puede tener una estructura autorregresiva en la que la heterocedasticidad observada a lo largo de diferentes periodos quizá esté autocorrelacionada. Así las tres características mencionadas de los rendimientos son precisamente por las cuales se diseñó dicho modelo. Bollerslev (1986), desarrolló el modelo GARCH el cual básicamente generaliza el modelo $A R C H$ que es puramente autorregresivo, para lograr un modelo autorregresivo de medias móviles. Se establece el supuesto de que las ponderaciones de los cuadrados de los residuos pasados disminuyen geométricamente a una tasa que debe estimarse a partir de los datos. Engle (2004) señala que la especificación CARCH $(1,1)$ es el caballo de batalla de las aplicaciones financieras, pues el modelo se puede emplear para describir la dinámica de volatilidad de casi todas las series de rendimientos financieros:

La predicción CARCH de la varianza es una media ponderada de tres predicciones diferentes de la varianza: una de ellas es una varianza constante que 
corresponde a la media de largo plazo; la segunda es la predicción que se hizo en el periodo anterior y la tercera corresponde a la nueva información que no estaba disponible cuando se hizo la predicción anterior (Engle,2004, p.225).

De este modo, el modelo GARCH para modelar la volatilidad queda establecido de la siguiente forma de acuerdo con Catalán (2011) y Martínez y Venegas (2014):

$$
\varepsilon_{\mathrm{t}} \rightarrow \mathrm{N}\left(0, \mathrm{y}_{\mathrm{t}}\right) \quad \varepsilon_{\mathrm{t}}=\sqrt{ }_{\mathrm{h}_{\mathrm{t}}} \mathrm{v}_{\mathrm{t}} \quad \mathrm{v}_{\mathrm{t}} \sim \mathrm{iidN}(0,1),
$$

así, todas las perturbaciones de $t$ a $\varepsilon_{t-q}$ tienen un efecto directo sobre $\varepsilon_{t^{\prime}}$ de tal for $\neg$ ma que la varianza condicional actúa como un proceso autorregresivo de orden q. Por lo tanto, la ecuación de la varianza del modelo GARCH $(1,1)$ está determinada según Bollerslev (1986) por:

$$
\sigma_{t}^{2}=\alpha_{0}+\sum_{\mathrm{i}=1}^{\mathrm{q}} \mathrm{a}_{\mathrm{i}} \varepsilon_{\mathrm{t}-1}^{2}+\sum_{\mathrm{i}=1}^{\mathrm{p}} \beta_{1} \mathrm{~h}_{\mathrm{t}-\mathrm{i}}
$$

Donde:

$\sigma_{t}^{2}$ es la varianza del error, $\varepsilon_{\mathrm{t}}$ es el proceso del error que se modelará y es condicional a la información disponible en el tiempo $t$, $\sum_{\mathrm{i}=1} \beta_{1} \mathrm{~h}_{\mathrm{t}-\mathrm{i}}$ es la varianza rezagada y $\sum_{\mathrm{i}=1} a_{\mathrm{i}} \varepsilon_{\mathrm{t}-\mathrm{i}}^{2}$ el shock de las noticias

Asimismo, las restricciones en el modelo son: $p \geq 0, q>0, a_{0}>0$ y $a_{1} \geq 0$ (para $\left.i=1,2, \ldots, q\right)$ y $\beta_{1} \geq 0$, (para $i=1,2, \ldots, p$.); es decir, el modelo requiere que la varianza condicional sea no negativa, entonces, la varianza no crece al infinito y describe un proceso estacionario dado por: $\Sigma_{\mathrm{i}=1}\left(a_{i} \beta_{1}<1\right)$. De esta manera el modelo GARCH permite modelar la varianza condicional como resultado de las varianzas condicionales pasadas; es decir, por los valores pasados de sí misma y por las perturbaciones pasadas.

\section{Análisis empírico}

El análisis inició con la estimación de los rendimientos tomando como base los precios de cierre diarios de las Siefores registrados en la Bolsa Mexicana de Valores. 
El periodo de estudio comprendió del 3 de marzo de 2008 (inicio de operaciones de la SB3) al 28 de junio de 2019. La muestra incluyó un total de diez Sociedades que cotizaron sin interrupción durante el periodo de estudio: Azteca, Citibanamex, Coppel, Inbursa, Invercap, PensionISSSTE, Principal, ProfuturoCNP, Sura y XXI Banorte. El horizonte de tiempo fue 135 meses (11.2 años) que representaron un total de 2846 datos diarios por cada fondo 4 .

A continuación, se presenta el procedimiento econométrico para la estimación de los rendimientos y el riesgo de mercado para dos fondos: La Siefore Azteca Básica de Pensiones, S.A. de C.V. (en adelante, Azteca) y la Siefore Citibanamex Básica de Pensiones, S.A. de C.V. (en adelante, Citibanamex). Cabe señalar que el mismo procedimiento fue aplicado para los ocho fondos restantes. El proceso de generación de datos basado en las series de tiempo busca ser estacionario, entonces, se crearon las variables de precios de Azteca y Citibanamex. Posteriormente se procedió a realizar una primera verificación tentativa de la existencia de estacionariedad bajo un análisis gráfico de las series en forma de niveles diarios.

GrÁFICA 6. Serie de PRECIOS PARA AZTECA: 2008-2019*

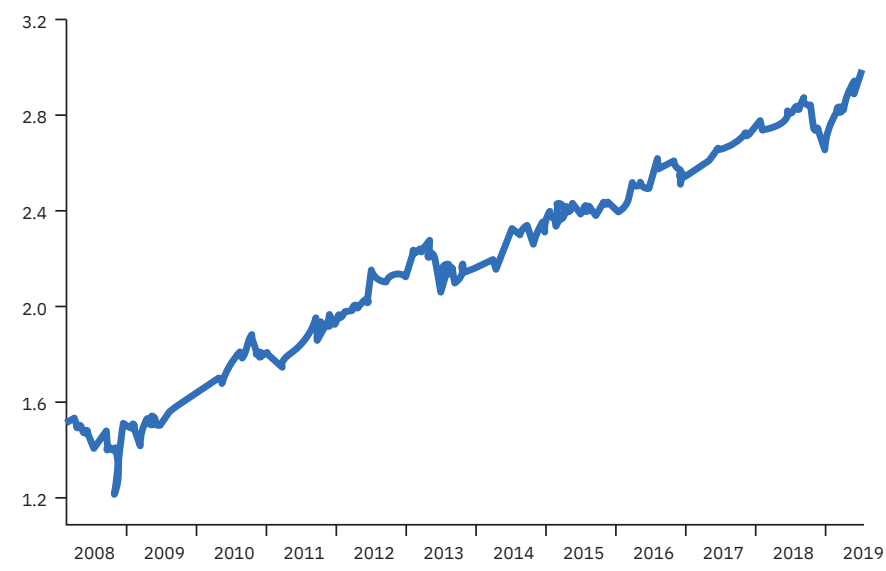

* Último dato al cierre de septiembre.

Fuente: Elaboración propia

\footnotetext{
${ }^{4}$ Se eliminaron los días 18 de noviembre de 2008 y 4 febrero de 2014 ya que la CONSAR repitió el precio del día anterior.
} 
Gráfica 7. Serie de precios para CitibanameX: 2008-2019*

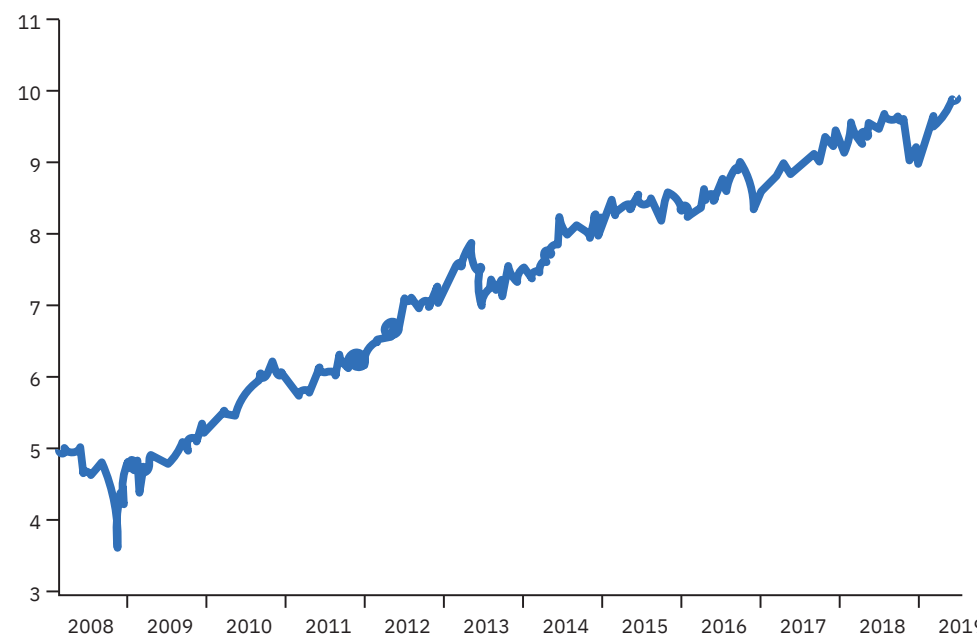

* Último dato al cierre de septiembre.

Fuente: Elaboración propia

Las gráficas anteriores muestran la evolución de los precios a lo largo de los últimos 11 años. Existe un componente de tendencia creciente (cambio del valor medio de la serie con el paso del tiempo) ya que a principios de 2008 , el precio registrado de Azteca fue 1.518674 y para 2019 se situó en 2.847326 (un crecimiento aproximado del 87\%). Por su parte, Citibanamex inicio con un precio de 5.000002 y cerró en 10.009901 . Es notoria la caída a valores mínimos a finales de 2008, en adelante se aprecian ligeros descensos entre 2011-2013 y una recuperación gradual con pocas fluctuaciones en los últimos dos años. Ambas series son no estacionarias, dado que no tienen la característica de media y varianza constante a lo largo de toda la muestra. Como se explicó, las series estacionarias son aquellas que resultan de la diferencia de los logaritmos que justamente coinciden con la serie de los rendimientos de un activo financiero. Entonces se procedió a generar variables del logaritmo de la serie original y posteriormente, se obtuvieron los rendimientos continuos de acuerdo con la ecuación (1.1) donde por simplificación, se asume que los pagos $D t$ son cero. Después, se graficaron las nuevas series para analizar el comportamiento de los rendimientos y verificar si cumplían o no con un proceso estacionario. 
GRÁFICA 8. SERIE DE PRECIOS PARA AZTECA: 2008-2019*

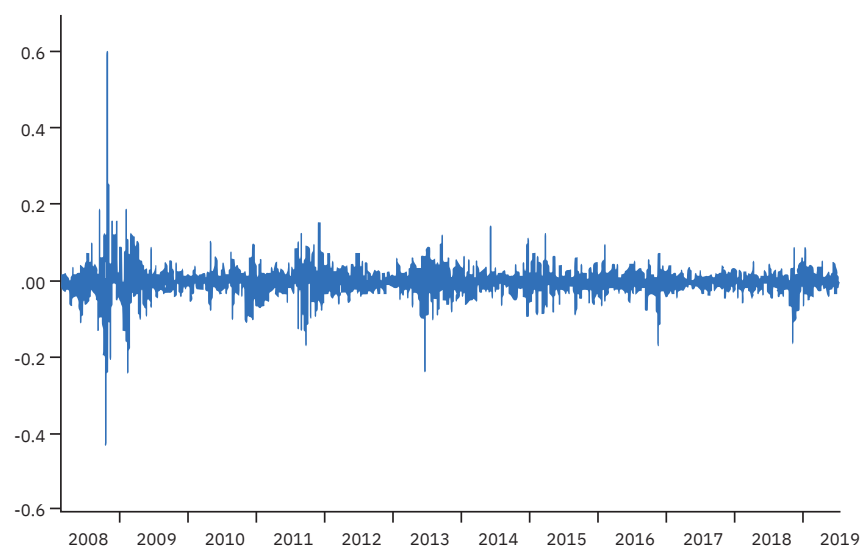

* Último dato al cierre de septiembre.

Fuente: elaboración propia

GrÁfica 9. Serie de precios para CitibanAMEX: 2008-2019*

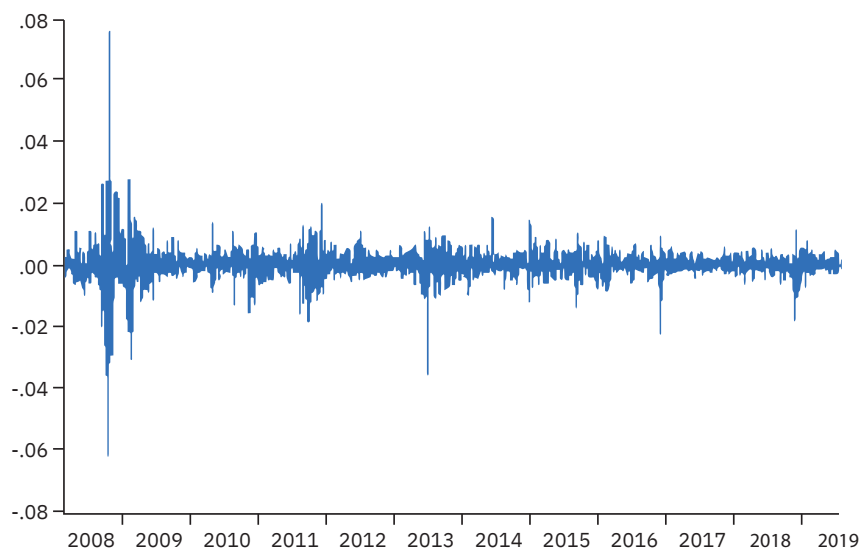

* Último dato al cierre de septiembre.

Fuente: elaboración propia

En las gráficas anteriores, las series de rendimientos están centradas en cero a lo largo del periodo de la muestra. Se observa en 2008 una altísima volatilidad de precios con una caída jamás vista en los últimos 11 años. Los precios fueron 
afectados por la crisis financiera internacional 2008-2009 que puso de manifiesto la vulnerabilidad de los sistemas financieros internacionales (sobre todo en países desarrollados) y en el caso mexicano, se manifestó (entre otros aspectos) en la caída de los fondos de pensiones. En adelante, se aprecia una nueva fase de volatilidad para 2011 explicada por la amenaza de que Grecia, Portugal, Irlanda y España incumplieran sus compromisos de deuda soberana (Santillán, Martínezy López, 2016). Hacia 2013 se aprecia una recuperación gradual con pocas fluctuaciones y a finales de 2018, la cancelación del Nuevo Aeropuerto Internacional de la Ciudad de México (NAICM), provocó un nuevo periodo de relativa volatilidad. De esta manera se evidenció que tanto eventos externos como internos de la economía nacional repercutieron de forma negativa en los precios de los fondos de pensiones en México.

Aunado a lo anterior, se observan clústers de volatilidad: la volatilidad alta en un periodo continuó siéndolo en el periodo siguiente, por el contrario, la que fue baja en un periodo, continuó siendo baja en el periodo siguiente. Los puntos donde la cotización de los precios disminuyó bastante, coincidió con los puntos donde la serie de rendimientos fue más volátil. De esta manera, la transformación de los precios a rendimientos permitió manejar información estable y más adecuada para la modelación econométrica. Ahora bien, para comprobar la normalidad de la distribución de los rendimientos se presentaron los siguientes gráficas referidas a histogramas con sus respectivos estadísticos básicos:

\section{GráfICA 10. HISTOGRAMA Y ESTADísticos báSICOS DE LOS RENDIMIENTOS DE AZTECA}

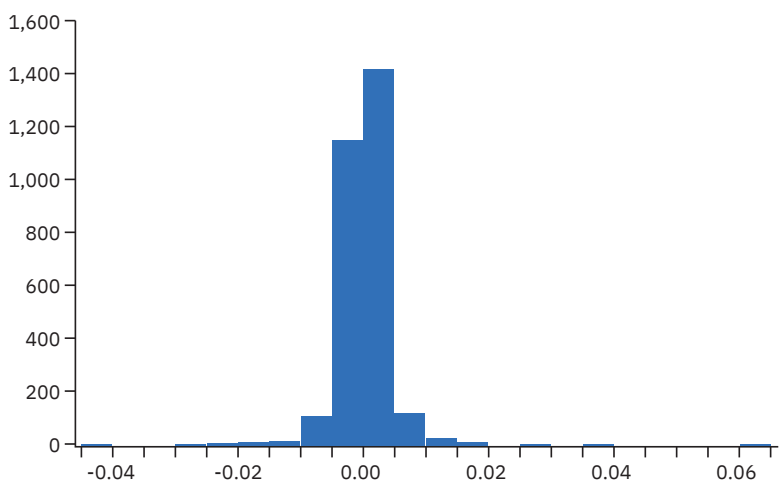

\begin{tabular}{|c|c|}
\hline \multicolumn{2}{|c|}{$\begin{array}{l}\text { Series: DLPAZT } \\
\text { Sample 3/03/2008 6/28/20 } \\
\text { Observations } 2846\end{array}$} \\
\hline \multicolumn{2}{|c|}{ Mean $\quad 0.000235$} \\
\hline Median & 0.000272 \\
\hline Maximum & 0.060676 \\
\hline Minimum & -0.042608 \\
\hline Std. Dev. & 0.003846 \\
\hline Skewness & 0.864374 \\
\hline Kurtosis & 38.56163 \\
\hline \multirow{2}{*}{$\begin{array}{l}\text { Jarque-Bera } \\
\text { Probability }\end{array}$} & 150318.4 \\
\hline & 0.000000 \\
\hline
\end{tabular}

Fuente: elaboración propia 
GRÁFICA 11. HISTOGRAMA Y ESTAdísticos bÁSICOS DE LOS RENDIMIENTOS DE CITIBANAMEX

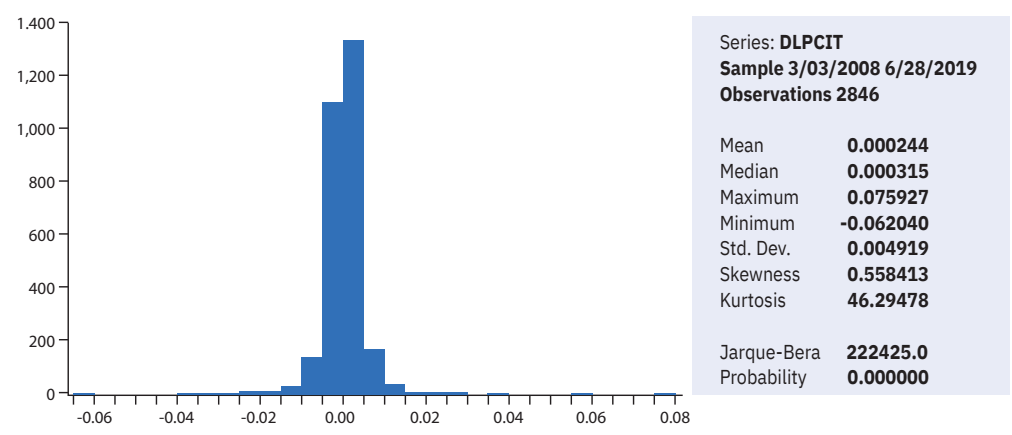

Fuente: elaboración propia

Al analizar los datos anteriores, se observa que la media se aproxima a cero para ambos fondos, se situó alrededor del $0.02 \%$ por día de apertura. El valor de la Kur-

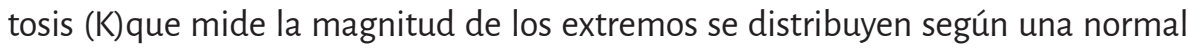
cuando $K=3$, pero cuanto menor a 3 (ya sea en valor superior o inferior) más se distancia de la forma de una función de distribución normal. El valor de K para Azteca fue 38.56163 y para Citibanamex de 46.29478 , dichos valores tan altos corresponden a distribuciones leptocúrticas e infieren la presencia de colas pesadas.

Además, ambos fondos, presentan coeficientes de asimetría (skewness) positivos, lo que indica que los rendimientos poseen asimetría en los extremos de la cola derecha con relación a una distribución normal. Por su parte, los valores del estadístico Jarque-Bera establecen la Hipótesis nula $\left(\mathrm{H}_{\mathrm{o}}\right)$ que las series poseen una distribución de probabilidad normal; al respecto, con un nivel de significancia de $5 \%$ y 2 grados de libertad para los rendimientos se obtuvo un valor Chi teórico de 5.992, por lo que se rechaza la $\mathrm{H}_{\circ}$ establecida por el valor Jarque-Bera (el cual fue de 150318.4 para Azteca y 222425.o para Citibanamex) por lo tanto, las series no responden a una distribución normal.

Con el objetivo de observar la factibilidad de modelar los rendimientos (las primeras diferencias de los logaritmos de los precios) bajo los Modelos ARMA, se realizaron dos pruebas formales de raíz unitaria para comprobar que las series fueran estacionarias: el Augmented Dickey-Fuller (ADF) y la prueba Phillips-Perron (PP). Estas pruebas establecen la $\mathrm{H}_{0}$ de presencia de raíz unitaria de acuerdo a la comparación entre el T-statistic y los distintos valores críticos de MacKinnon. 
Se dice que una serie presenta raíz unitaria si alguno de dichos valores es mayor que el T-statistic (en valores absolutos). Por lo tanto, se procedió a efectuar ambas pruebas y los resultados se muestran en los dos siguientes cuadros:

\section{Cuadro 4. Pruebas de raíz unitaria ADF y PP para Azteca}

\begin{tabular}{|c|c|c|c|c|c|c|c|c|}
\hline \multirow{2}{*}{ Variable } & \multirow{2}{*}{ Modelo } & \multicolumn{7}{|c|}{ Pruebas } \\
\hline & & ADF & P-value & DW & PP & P-value & DW & Clasificación \\
\hline \multirow{3}{*}{ T-statistic } & $1 \mathrm{ADFN}$ & -34.1831 & 0.0000 & 2.00327 & -39.55658 & 0.0000 & 1.96812 & $\begin{array}{l}\text { sin raíz } \\
\text { unitaria }\end{array}$ \\
\hline & 2 ADFI & -34.3182 & 0.0000 & 2.00371 & -39.4559 & 0.0000 & 1.96725 & $\begin{array}{l}\text { sin raíz } \\
\text { unitaria }\end{array}$ \\
\hline & 3 ADIT & -34.3121 & 0.0000 & 2.00371 & -39.4476 & 0.0000 & 1.96725 & $\begin{array}{l}\text { sin raíz } \\
\text { unitaria }\end{array}$ \\
\hline \multirow{3}{*}{$\begin{array}{l}\text { Valores } \\
\text { críticos de } \\
\text { MacKinnon } \\
\text { al } 5 \%\end{array}$} & $1 \mathrm{ADFN}$ & $-3.41139 *$ & & & $-1.940935^{\star}$ & & & \\
\hline & 2 ADFI & $-1.94094^{*}$ & & & $-2.862357^{\star}$ & & & \\
\hline & 3 ADFTI & $-3.41139^{*}$ & & & $-3.411390^{*}$ & & & \\
\hline
\end{tabular}

* Indica que la prueba fue significativa al 95\%. Para llevar a cabo el contraste de la estacionariedad se aplicaron las pruebas ADF (Augmented Dickey-Fuller) y PP (Phillips-Perron) para los siguientes tres modelos: 1 ) sin intercepto y sin tendencia, 2) con intercepto o deriva y 3 ) con intercepto y con tendencia determinística y/o estocástica.

Fuente: elaboración propia

\section{Cuadro 5. Pruebas de Raíz Unitaria ADF y PP para CitibanameX}

\begin{tabular}{lcccccccc}
\hline \multirow{2}{*}{ Variable } & Modelo & \multicolumn{7}{c}{ Pruebas } \\
\cline { 2 - 8 } & & ADF & P-value & DW & PP & P-value & DW & Clasificación \\
\hline & 1 ADFN & -33.9483 & 0.0000 & 2.0054 & -38.51836 & 0.0000 & 1.96366 & $\begin{array}{c}\text { sin raíz } \\
\text { unitaria }\end{array}$ \\
\cline { 2 - 8 } T-statistic & 2 ADFI & -34.0335 & 0.0000 & 2.00574 & -38.49094 & 0.0000 & 1.96307 & $\begin{array}{l}\text { sin raíz } \\
\text { unitaria }\end{array}$ \\
\cline { 2 - 8 } & 3 ADIT & -34.0278 & 0.0000 & 2.00574 & -38.48232 & 0.0000 & 1.96306 & $\begin{array}{l}\text { sin raíz } \\
\text { unitaria }\end{array}$ \\
\hline $\begin{array}{l}\text { Valores } \\
\text { críticos de } \\
\begin{array}{l}\text { MacKinnon } \\
\text { al 5\% }\end{array}\end{array}$ & 1 ADFN & -1.94094 & & & -1.940935 & & \\
\cline { 2 - 8 } & 2 ADFI & -2.86236 & & -2.862357 & & \\
\hline
\end{tabular}

* Indica que la prueba fue significativa al 95\%. Para llevar a cabo el contraste de la estacionariedad se aplicaron las pruebas ADF (Augmented Dickey-Fuller) y PP (Phillips-Perron) para los siguientes tres modelos: 1) sin intercepto y sin tendencia, 2) con intercepto o deriva y 3) con intercepto y con tendencia determinística y/o estocástica.

Fuente: elaboración propia 
Para Azteca y Citibanamex, se observó que al 95\% de confianza se rechazó la $\mathrm{H}_{\circ}$ de presencia de raíz unitaria después de aplicar las primeras diferencias. Los distintos valores de los T-statistic fueron mayores que cualquiera de los valores críticos de MacKinnon, por lo tanto, las series fueron estacionarias. Además, en el test Durbin-Watson los rezagos incorporados en las pruebas ADFy PP eliminaron correctamente posibles problemas de autocorrelación en los residuos.

En el análisis de las series se incluyó una tercera prueba de raíz unitaria llamada Kwiatkowski-Phillips-Schmidt-Shin (KPSS) que establece la $\mathrm{H}_{0}$ de presencia de estacionariedad de acuerdo con la comparación entre el KPSS-statistic y los distintos valores críticos asintóticos. Una serie es estacionaria si alguno de dichos valores es mayor que el KPSS-statistic (en valores absolutos). Los resultados de la prueba aplicada a las primeras diferencias de los logaritmos se presentan en los siguientes dos cuadros, donde se rechazó la $\mathrm{H}_{\mathrm{o}}$; por tanto, las series no fueron estacionarias, lo que indicó la existencia de raíces unitarias, contradiciendo los resultados de las pruebas ADF y PP realizadas anteriormente.

Cuadro 6. Prueba de Raíz unitaria KPSS para Azteca

\begin{tabular}{lllll}
\hline \multirow{2}{*}{ Variable } & Modelo & \multicolumn{3}{c}{ Prueba } \\
\cline { 2 - 5 } & & KPSS & DW & Clasificación \\
\hline \multirow{2}{*}{ T-statistic } & 1 KPSSI & 0.036964 & 1.467324 & con raíz unitaria \\
\cline { 2 - 4 } & 2 KPSSTI & 0.035731 & 1.467325 & con raíz unitaria \\
\hline $\begin{array}{l}\text { Valores críticos asintóticos al } \\
5 \%\end{array}$ & 1 KPSSI & 0.463000 & \\
\cline { 2 - 4 } & 2 KPSSTI & 0.146000 & \\
\hline
\end{tabular}

Fuente: elaboración propia

Cuadro 7. Prueba de Raíz unitaria KPSS para CitibanameX

\begin{tabular}{lllll}
\hline \multirow{2}{*}{ Variable } & Modelo & \multicolumn{3}{c}{ Prueba } \\
\cline { 2 - 5 } & & KPSS & DW & Clasificación \\
\hline \multirow{2}{*}{ T-statistic } & 1 KPSSI & 0.049204 & 1.431919 & con raíz unitaria \\
\cline { 2 - 5 } & 2 KPSSTI & 0.042079 & 1.431932 & con raíz unitaria \\
\hline \multirow{2}{*}{ Valores críticos asintóticos al 5\% } & 1 KPSSI & 0.463000 & \\
\cline { 2 - 4 } & 2 KPSSTI & 0.146000 & \\
\hline
\end{tabular}

Fuente: elaboración propia 
Al detectar la inconsistencia entre las pruebas de raíces unitarias, es probable que las series presentaran un rompimiento o cambio estructural, el cual significa que los valores de los parámetros del modelo no permanecen constantes a lo largo de todo el periodo (Gujarati y Porter (2010). En el caso de las Siefores, es importante mencionar que en el amplio periodo de análisis de esta investigación (11.2 años) pudieron existir cambios estructurales debido a factores externos (por ejemplo, la crisis subprime 2008-2009, la crisis de deuda soberana de los países europeos en 2011 o bien las decisiones de políticas económicas internas (por ejemplo, la cancelación del NAICM. Los argumentos anteriores se respaldaron a través de las Gráficas 10 y 11, las cuales evidenciaron la existencia de periodos de alta volatilidad que impactaron de manera negativa el comportamiento de los rendimientos de las Sociedades. Para conocer si efectivamente ocurrió o no un cambio estructural se aplicó la prueba Phillips-Perron (PP) con raíz unitaria y con rompimiento estructural para los dos fondos que arrojó los siguientes resultados:

\section{Gráfica 12. Prueba de RAíz UNITARIA PP CON PRESENCIA}

\section{DE ROMPIMIENTO ESTRUCTURAL PARA AZTECA}

\section{Perron Breakpoint}

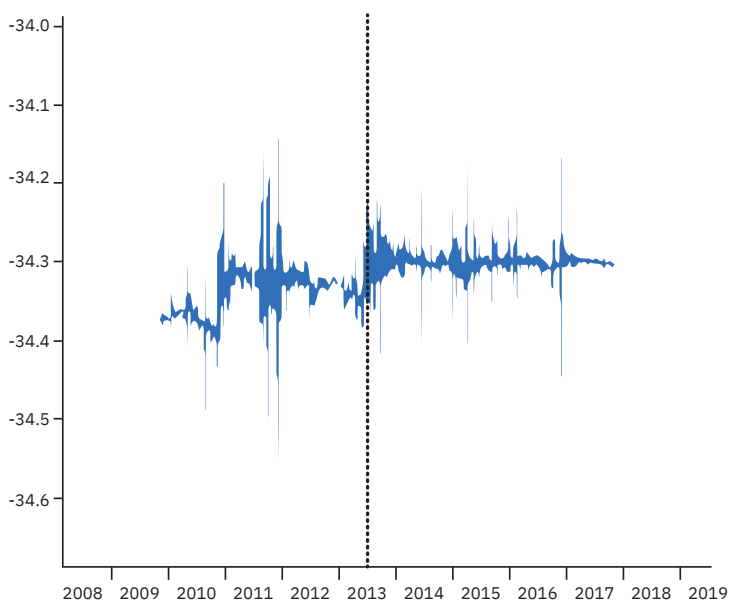

Muestra: 3/03/2008 6/28/2019

Observaciones efectivas: 2847

Hipótesis nula $(\mathrm{HO})$ : la variable DLPAZT tiene

raiz unitaria con rompimiento estructural

tanto en tendencia como en intercepto.

Longitud de rezago elegido: 1 (Rezago máximo: 1 )

Fecha de rompimiento estructural: 6/21/2013

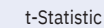

Prueba de Perron para raíz unitaria: $\quad-34.68480$

$1 \%$ valor crítico:

$5 \%$ valor crítico:

$10 \%$ valor crítico: 
GráfICA 11. PRUEBA de RAíz UNITARIA PP CON PRESENCIA DE ROMPIMIENTO ESTRUCTURAL PARA CitibanameX

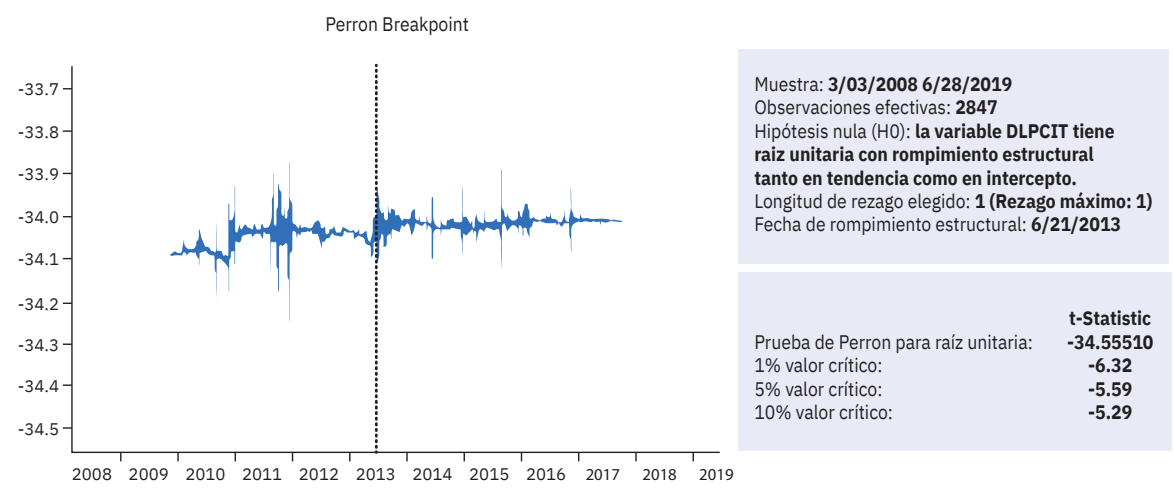

Fuente: Elaboración propia

Las pruebas anteriores evidenciaron un claro rompimiento estructural para los dos fondos que ocurrió el día 21 de junio de 2013. Considerando lo anterior se procedió a crear variables "Dummy" o variables dicotómicas que consideraron las modalidades de ausencia/presencia (0/1) en la regresión de los modelos propuestos para cada Siefore. Así, la "Dummy" fue nombrada como (D13) para incorporar el cambio estructural en la modelación de las series de los rendimientos de Azteca y Citibanamex. Por lo tanto, D13 se creó con valores de "cero" en todas las observaciones, excepto el 21 de junio de 2013, donde se le asignó el valor "uno", respectivamente.

Para identificar los valores apropiados de $p, d, y$ q se aplicó la metodología Box-Jenkins identificando inicialmente el modelo a estimar. Para ello se utilizó el análisis gráfico de los correlogramas de los residuos al cuadrado de las series analizadas que proporcionaron indicios para poder estimar que las dos series siguieron un proceso AR puro (el valor de $p$ ) bajo Modelos Autorregresivos de orden "uno" o AR(1) y un valor de Medias Móviles (el valor de q) de "once" o MA(11) ya que el primer y onceavo rezago de la Función de Autocorrelación (FAC) y la Función de Autocorrelación Parcial (FACP) presentaron el patrón típico de pico. Respecto a la incorporación de las variables "Dummy" en el modelaje de las series, en el siguiente cuadro se comprobó que el valor del estadístico de significancia del coeficiente D13, resultó significativo a menos del 1\%, indicando que el cambio estructural detectado fue muy significativo en el modelo ARMA de las dos Siefores. 
Riesgo y rendimiento de los fondos de pensiones en México: Análisis de la SIEFORE básica 3

Cuadro 8. Modelos ARMA estimados para Azteca y CitibanameX

\begin{tabular}{lrr}
\hline Parámetros & \multicolumn{1}{c}{ Azteca } & Citibanamex \\
\hline$\alpha 0$ & AR (1) MA(11) & AR (1) MA(11) \\
\hline$z$-Statistic & 0.000324 & 0.000378 \\
\hline P-value & 7.383735 & 6.863967 \\
\hline$\alpha 1$ & 0.00000 & 0.00000 \\
\hline Z-stat & 0.12168 & 0.15467 \\
\hline P-value & 5.939527 & 6.902498 \\
\hline$\alpha 2$ & 0.00000 & 0.00000 \\
\hline Z-stat & -0.053729 & -0.062835 \\
\hline$P$ & -2.816685 & -3.306283 \\
\hline D13 (Dummy) & 0.0049 & 0.0009 \\
\hline Z-stat & -0.023902 & -0.037575 \\
\hline P-value & -26.09047 & -15.10759 \\
\hline
\end{tabular}

Fuente: elaboración propia

Al ser modelos integrados de orden 1, por sus primeras diferencias dadas por I(0) fueron estacionarios. La búsqueda de estacionariedad de las series coincidió con la estimación de los rendimientos diarios para un horizonte de los últimos 135 meses. Posteriormente, se estimó el rendimiento promedio anualizado de acuerdo con la ecuación (2). De esta manera, en el siguiente cuadro se presentan los rendimientos de las Siefores de mayor a menor desempeño.

\section{CuAdRo 9. RENDIMIENTO PROMEDIO ANUALIZADO DE LAS 10}

SIEFORE DE LA SB3: 2008-2018 -ÚLTIMOS 135 MESES-

\begin{tabular}{llc}
\hline & Siefore & $\begin{array}{c}\text { Rendimiento promedio } \\
\text { anualizado* }\end{array}$ \\
\hline 1 & PensionISSSTEt & $7.41 \%$ \\
\hline 2 & ProfuturoGNP & $6.97 \%$ \\
\hline 3 & Sura & $6.90 \%$ \\
\hline 4 & Citibanamex & $6.15 \%$ \\
\hline 5 & XXI Banorte & $6.11 \%$ \\
\hline 7 & Azteca & $5.92 \%$ \\
\hline 8 & Principal & $5.84 \%$ \\
\hline 9 & Inbursa & $5.78 \%$ \\
\hline 10 & Invercap & $5.56 \%$ \\
\hline & Promedio simple & $4.76 \%$ \\
\hline
\end{tabular}

* El rendimiento diario se anualizó multiplicándolo por la raíz del número de días que las SB3 cotizó en el año (252.33 días) para obtener la tasa promedio anualizada. $t$ Este fondo inició operaciones a partir de diciembre de 2008, por lo que el cálculo fue estimado del 4 de diciembre de 2008 al 28 de junio de 2019.

Fuente: Elaboración propia con datos de la CONSAR 
Una vez estimados los rendimientos, se procedió a calcular el riesgo de mercado, procediendo a la varianza condicional de ambas Siefores con los modelos de la familia ARIMA-CARCH. El Modelo GARCH se compone de dos ecuaciones interrelacionadas: una para la media condicional que se indica aquí como un Modelo ARIMA $(p .1, q)$ que se presenta en la primera parte de los cuadros 10 y 11 y la ecuación de la varianza condicional $\left(\sigma_{t}^{2}\right)$ dada por la ecuación 3 que aparece en la segunda parte de dichos cuadros5. Ambas ecuaciones se resuelven de manera simultánea para obtener los coeficientes y verificar la significancia estadística de cada uno y así seleccionar el modelo con mejor ajuste. Para comprobar el efecto ARCH, se utilizó la prueba del Multiplicador de Lagrange que se basa en la autocorrelación de los residuos elevados al cuadrado y en ambos casos se rechazó la Ho de ausencia de efectos de heteroscedasticidad condicional autorregresiva con una significancia menor al 1\%. Por su parte, el parámetro sujeto a la regresión fue inferior a 1 (para Azteca 0.447507 y para Citibanamex 0.466425) confirmando que las series si tenían efectos $\mathrm{ARCH}$ y por consiguiente la varianza de los rendimientos fue variante en el tiempo y pudo ser modelada. Considerando lo anterior, se estimaron cuatro modelos para cada Siefore y a continuación se presentan los resultados de las estimaciones de los modelos ARIMA-CARCH para Azteca y Citibanamex.

CuAdro 10. Modelos ARIMA-GARCH estimados PARA AZTECA

\begin{tabular}{lcccc}
\hline \multicolumn{4}{c}{ Modelos } \\
\hline Parámetros & AR (1) MA(11) D13 & AR (1) MA(11) D13 & AR (1) MA(11) D13 & AR (1) MA(11) D13 \\
\hline$\alpha 0$ & 0.000324 & 0.000328 & 0.000329 & 0.000326 \\
\hline Z-stat & 7.383735 & 7.435252 & 7.465413 & 7.406141 \\
\hline P-value & 0.0000 & 0.0000 & 0.0000 & 0.0000 \\
\hline$\alpha 1$ & 0.12168 & 0.122262 & 0.121917 & 0.122127 \\
\hline Z-stat & 5.939527 & 5.946128 & 5.922182 & 5.941307 \\
\hline P-value & 0.0000 & 0.0000 & 0.0000 & 0.0000 \\
\hline$\alpha 2$ & -0.053729 & -0.052712 & -0.052546 & -0.052944 \\
\hline
\end{tabular}

\footnotetext{
${ }^{5}$ La forma de presentar parte de los resultados de los modelos ARIMA-GARCH estimados en este trabajo toma como referencia la investigación de Santillán, Martínez y López (2016).
} 
Riesgo y rendimiento de los fondos de pensiones en México: Análisis de la SIEFORE básica 3

Cuadro 10. Modelos ARIMA-GARCH estimados para AzteCA (CONTINUACión)

\begin{tabular}{|c|c|c|c|c|}
\hline \multirow[b]{2}{*}{ Z-stat } & \multicolumn{4}{|c|}{ Modelos } \\
\hline & -2.816685 & -2.761198 & -2.756522 & -2.771751 \\
\hline P-value & 0.0049 & 0.0058 & 0.0058 & 0.0056 \\
\hline $\begin{array}{l}\text { D13 (Dum- } \\
\text { my)* }\end{array}$ & -0.023902 & -0.023894 & -0.023891 & -0.023919 \\
\hline Z-stat & -26.09047 & -26.9404 & -27.07134 & -26.15308 \\
\hline \multirow[t]{2}{*}{$\mathrm{P}$-value } & 0.0000 & 0.0000 & 0.0000 & 0.0000 \\
\hline & GARCH $(1,1)$ & GARCH $(2,1)$ & GARCH $(1,2)$ & GARCH $(2,2)$ \\
\hline$\alpha 0$ & $2.42 \mathrm{E}-07$ & $2.65 \mathrm{E}-07$ & $2.11 \mathrm{E}-07$ & $3.08 \mathrm{E}-07$ \\
\hline Z-stat & 3.8056 & $3.80 E+00$ & $3.01 E+00$ & 7.88E-01 \\
\hline P-value & 0.0001 & 0.0001 & 0.0026 & 0.4307 \\
\hline$\alpha 1$ & 0.149195 & 0.126413 & 0.125871 & 0.133796 \\
\hline Z-stat & 7.207371 & 3.658827 & 4.143949 & 3.864996 \\
\hline P-value & 0.0000 & 0.0003 & 0.0000 & 0.0001 \\
\hline$\beta 1$ & 0.833788 & 0.033529 & 1.060402 & 0.053819 \\
\hline Z-stat & 43.73473 & 0.858471 & 5.089821 & 0.231598 \\
\hline P-value & 0.0000 & 0.3906 & 0.0000 & 0.8169 \\
\hline$\beta 2$ & & 0.821441 & -0.200996 & 0.608885 \\
\hline Z-stat & & 35.94604 & -1.123294 & 0.384776 \\
\hline P-value & & 0.0000 & 0.2613 & 0.7004 \\
\hline$\beta 3$ & & & & 0.181729 \\
\hline Z-stat & & & & 0.13764 \\
\hline P-value & & & & 0.8905 \\
\hline $\begin{array}{l}\text { Akaike info } \\
\text { criterion }\end{array}$ & -8.921386 & -8.921094 & -8.92122 & -8.920148 \\
\hline $\begin{array}{l}\text { Schwarz } \\
\text { criterion }\end{array}$ & -8.921094 & -8.904353 & -8.904479 & -8.901315 \\
\hline $\begin{array}{l}\text { Han- } \\
\text { nan-Quinn } \\
\text { criter. }\end{array}$ & -8.904353 & -8.915056 & -8.915182 & -8.913356 \\
\hline
\end{tabular}

* De acuerdo con la prueba Phillps-Perron con rompimiento estructural se incorporó la variable Dummy el día 21 de junio de 2013.

Fuente: elaboración propia 
Raúl Arturo Cornejo López (†) • Israel Bermúdez Pacheco

Cuadro 11. Modelos ARIMA-GARCH estimados PARA CitibanameX

\begin{tabular}{|c|c|c|c|c|}
\hline \multirow[b]{2}{*}{ Parámetros } & \multicolumn{4}{|c|}{ Modelos } \\
\hline & AR (1) MA(11) D13 & AR (1) MA(11) D13 & AR (1) MA(11) D13 & AR (1) MA(11) D13 \\
\hline$\alpha 0$ & 0.000378 & 0.000379 & 0.000383 & 0.000383 \\
\hline Z-stat & 6.863967 & 6.863811 & 6.981429 & 6.992685 \\
\hline P-value & 0.0000 & 0.0000 & 0.0000 & 0.0000 \\
\hline$\alpha 1$ & 0.154667 & 0.158016 & 0.159243 & 0.158774 \\
\hline Z-stat & 6.902498 & 7.190078 & 7.358852 & 7.332659 \\
\hline$P$-value & 0.0000 & 0.0000 & 0.0000 & 0.0000 \\
\hline$\alpha 2$ & -0.062835 & -0.060271 & -0.058891 & -0.059236 \\
\hline Z-stat & -3.306283 & -3.163446 & -3.101078 & -3.123391 \\
\hline P-value & 0.0009 & 0.0016 & 0.0019 & 0.0018 \\
\hline D13 (Dummy)* & -0.037575 & -0.038382 & -0.04005 & -0.040055 \\
\hline Z-stat & -15.10759 & -10.26995 & -7.049739 & -7.045217 \\
\hline \multirow[t]{2}{*}{ P-value } & 0.0000 & 0.0000 & 0.0000 & 0.0000 \\
\hline & GARCH $(1,1)$ & GARCH $(2,1)$ & GARCH $(1,2)$ & GARCH $(2,2)$ \\
\hline$\alpha 0$ & $3.41 E-07$ & $3.75 E-07$ & $2.69 \mathrm{E}-07$ & $2.51 E-07$ \\
\hline Z-stat & 3.962899 & $3.89 \mathrm{E}+00$ & $3.22 \mathrm{E}+00$ & $1.50 \mathrm{E}+00$ \\
\hline P-value & 0.0001 & 0.0001 & 0.0013 & 0.1341 \\
\hline$\alpha 1$ & 0.173638 & 0.141437 & 0.126856 & 0.131314 \\
\hline Z-stat & 7.167351 & 3.967406 & 4.623241 & 3.842162 \\
\hline P-value & 0.0000 & 0.0001 & 0.0000 & 0.0001 \\
\hline$\beta 1$ & 0.813968 & 0.042717 & 1.174795 & -0.012961 \\
\hline Z-stat & 40.04487 & 0.87949 & 5.835946 & -0.13605 \\
\hline$P$-value & 0.0000 & 0.3791 & 0.0000 & 0.8918 \\
\hline$\beta 2$ & & 0.801329 & -0.312323 & 1.219182 \\
\hline Z-stat & & 29.24847 & -1.79895 & 2.735505 \\
\hline$P$-value & & 0.0000 & 0.072 & 0.0062 \\
\hline$\beta 3$ & & & & -0.347385 \\
\hline
\end{tabular}


Riesgo y rendimiento de los fondos de pensiones en México: Análisis de la SIEFORE básica 3

Cuadro 11. Modelos ARIMA-GARCH estimados PARA CitibanameX (CONTINUACIÓN)

\begin{tabular}{|c|c|c|c|c|}
\hline & \multicolumn{4}{|c|}{ Modelos } \\
\hline \multicolumn{3}{|l|}{ Z-stat } & & -0.960966 \\
\hline \multicolumn{4}{|l|}{ P-value } & 0.3366 \\
\hline $\begin{array}{l}\text { Akaike info } \\
\text { criterion }\end{array}$ & -8.615569 & -8.615511 & -8.616181 & -8.615505 \\
\hline $\begin{array}{l}\text { Schwarz } \\
\text { criterion }\end{array}$ & -8.600921 & -8.598771 & -8.599441 & -8.596672 \\
\hline $\begin{array}{l}\text { Hannan-Quinn } \\
\text { criter. }\end{array}$ & -8.610285 & -8.609473 & -8.610143 & -8.608712 \\
\hline
\end{tabular}

* De acuerdo con la prueba Phillps-Perron con rompimiento estructural se incorporó la variable Dummy el día 21 de junio de 2013.

Fuente: elaboración propia

La estrategia de selección del modelo con mejor ajuste fue la comparación entre los criterios de información de Akaike, Schwarz y Hannan-Quinn (celdas en rojo). Se minimizó sin tomar en cuenta los valores absolutos; es decir, considerando los signos tal cual. De esta manera los resultados de las estimaciones de los modelos ARIMA-GARCH permitieron seleccionar que el modelo con mejor ajuste para Azteca y Citibanamex fue un AR(1) MA(11) D13 GARCH $(1,1)$ ya que en ambos casos, los valores del modelo fueron estadísticamente significativos, el coeficiente de la constante fue positivo y la suma de los coeficientes del término ARCH (el shock de las noticias) y del término $\mathrm{GARCH}$ (la volatilidad de un periodo anterior) fueron menores a 1 , lo que indica que los modelos fueron estacionarios. El siguiente cuadro presenta los modelos econométricos seleccionados para las diez Siefores analizadas, así como la ecuación de la varianza de las mismas.

CUAdRo 12. MODELOS ESTIMAdOS Y ECUACIONES DE VARIANZA PARA 10 SIEFORES DE LA SB3.

\begin{tabular}{|c|c|c|c|}
\hline & Siefore & Modelo & Ecuación de la varianza \\
\hline 1 & Azteca & $\begin{array}{l}\operatorname{AR}(1) \mathrm{MA}(11) \mathrm{D} 13 \\
\operatorname{GARCH}(1,1)\end{array}$ & $\sigma_{t}^{2}=2.42 E-07+0.149195 \varepsilon_{t-1}^{2}+0.833788 \sigma_{t-1}^{2}$ \\
\hline 2 & Citibanamex & $\begin{array}{l}\operatorname{AR}(1) \operatorname{MA}(11) \mathrm{D} 13 \\
\operatorname{GARCH}(1,1)\end{array}$ & $\sigma_{t}^{2}=3.41 E-07+0.173638 \varepsilon_{t-1}^{2}+0.813968 \sigma_{t-1}^{2}$ \\
\hline 3 & Coppel & $\begin{array}{l}\operatorname{AR}(1) M A(11) D 13 \\
\operatorname{GARCH}(1,1)\end{array}$ & $\sigma_{t}^{2}=2.09 E-07+0.138711 \varepsilon_{t-1}^{2}+0.840424 \sigma_{t-1}^{2}$ \\
\hline
\end{tabular}


CUADRO 12. MODELOS ESTIMADOS Y ECUACIONES DE VARIANZA PARA 10 SIEFORES DE LA SB3 (CONTINUACIÓN).

\begin{tabular}{|c|c|c|c|}
\hline & Siefore & Modelo & Ecuación de la varianza \\
\hline 4 & Inbursa & $\operatorname{AR}(1) \operatorname{MA}(8)$ GARCH $(1,1)$ & $\sigma_{t}^{2}=1.73 E-08+0.083363 \varepsilon_{t-1}^{2}+0.910812 \sigma_{t-1}^{2}$ \\
\hline 5 & Invercap & $\begin{array}{l}\operatorname{AR}(1) M A(1) \text { D08 } \\
\operatorname{GARCH}(1,1)\end{array}$ & $\sigma_{\mathrm{t}}^{2}=5.07 \mathrm{E}-08+0.141945 \varepsilon_{\mathrm{t}-1}^{2}+0.872306 \sigma_{\mathrm{t}-1}^{2}$ \\
\hline 6 & $\begin{array}{l}\text { Pensio- } \\
\text { nISSSTE }\end{array}$ & $\begin{array}{l}\operatorname{AR}(1) \mathrm{MA}(11) \mathrm{D} 16 \\
\operatorname{GARCH}(1,1)\end{array}$ & $\sigma_{t}^{2}=2.67 E-07+0.143202 \varepsilon_{t-1}^{2}+0.840326 \sigma_{t-1}^{2}$ \\
\hline 7 & Principal & $\begin{array}{l}\operatorname{AR}(1) \operatorname{AR}(11) \mathrm{D} 13 \\
\operatorname{GARCH}(1,2)\end{array}$ & $\sigma_{\mathrm{t}}^{2}=3.12 \mathrm{E}-07 .+0.153871 \varepsilon_{\mathrm{t}-1}^{2}+.0 .827724 \sigma_{\mathrm{t}-1}^{2}$ \\
\hline 8 & $\begin{array}{l}\text { Profuturo } \\
\text { GNP }\end{array}$ & $\operatorname{AR}(1) \operatorname{GARCH}(11) \mathrm{D} 10 \mathrm{GARCH}(1,1)$ & $\sigma_{t}^{2}=2.65 E-07+0.133158 \varepsilon_{t-1}^{2}+0.85683 \sigma_{t-1}^{2}$ \\
\hline 9 & Sura & $\begin{array}{l}\operatorname{AR}(1) \mathrm{MA}(11) \mathrm{D} 13 \\
\operatorname{GARCH}(1,1)\end{array}$ & $\sigma_{t}^{2}=5.99 E-08+0.16466 \varepsilon_{t-1}^{2}+0.833107 \sigma_{t-1}^{2}$ \\
\hline 10 & XXI Banorte & $\begin{array}{l}\text { AR(1) MA(1) D09 } \\
\text { GARCH }(1,1)\end{array}$ & $\sigma_{t}^{2}=3.95 E-07+0.493477 \varepsilon_{t-1}^{2}+0.647737 \sigma_{t-1}^{2}$ \\
\hline
\end{tabular}

Fuente: elaboración propia

Interpretando las ecuaciones de varianza, se observa que los valores y el parámetro asignado del GARCH $(1,1)$ fue estadísticamente significativo y la varianza fue positiva y finita para las diez Sociedades. Por su parte, la suma de los coeficientes de los términos ARCH y GARCH fueron 0.98298300 para Azteca y 0.98760600 para Citibanamex, ambos valores cercanos a la unidad significan que los shocks fueron bastante persistentes y que el proceso de varianza es convergente a su valor no condicional o de largo plazo. Una vez resueltas las ecuaciones de varianzas, se estimó el riesgo promedio anualizado y se presentaron los resultados en el siguiente cuadro de mayor a menor riesgo.

\section{CUADRO 13. RENDIMIENTO Y RIESGO PROMEDIO ANUALIZADO PARA 10 SIEFORE DE LA SB3:2008-2019 -ÚLTIMOS 135 MESES-}

\begin{tabular}{llcc}
\hline Siefore & $\begin{array}{c}\text { Rendimiento promedio } \\
\text { anualizado* }\end{array}$ & $\begin{array}{c}\text { Riesgo promedio } \\
\text { anualizado* }\end{array}$ \\
\hline 1 & PensionISSSTEt & $7.41 \%$ & $15.62 \%$ \\
\hline 2 & ProfuturoGNP & $6.97 \%$ & $15.73 \%$ \\
\hline
\end{tabular}


CUADRO 13. RENDIMIENTO Y RIESCO PROMEDIO ANUALIZADO PARA 10 SIEFORE DE LA SB3:2008-2019-ÚLTIMOS 135 MESES- (CONTINUACIÓN)

\begin{tabular}{llcc}
\hline Siefore & $\begin{array}{c}\text { Rendimiento promedio } \\
\text { anualizado* }\end{array}$ & $\begin{array}{c}\text { Riesgo promedio anuali- } \\
\text { zado* }\end{array}$ \\
\hline 3 & Sura & $6.90 \%$ & $15.85 \%$ \\
\hline 4 & Citibanamex & $6.15 \%$ & $15.69 \%$ \\
\hline 5 & XXI Banorte & $6.11 \%$ & $18.13 \%$ \\
\hline 6 & Azteca & $5.92 \%$ & $15.61 \%$ \\
\hline 7 & Principal & $5.84 \%$ & $15.59 \%$ \\
\hline 8 & Coppel & $5.78 \%$ & $15.55 \%$ \\
\hline 9 & Inbursa & $5.56 \%$ & $15.79 \%$ \\
\hline 10 & Invercap & $4.76 \%$ & $16.11 \%$ \\
\hline & Promedio simple SB3 & $6.14 \%$ & $15.97 \%$ \\
\hline
\end{tabular}

* El rendimiento y riesgo diario se anualizaron multiplicándolos por la raíz del número de días que las SB3 cotizó en el año (252.3 días) para obtener la tasa promedio anualizada.

$t$ Este fondo inició operaciones a partir de diciembre de 2008, por lo que el cálculo fue estimado del 4 de diciembre de 2008 al 28 de junio de 2019.

Fuente: elaboración propia

Una vez calculados los rendimientos y el riesgo, se procedió a comprobar la relación entre estas dos variables mediante la Gráfica 12, en la cual se colocaron los valores obtenidos del riesgo en abscisas, los rendimientos en ordenadas y la relación riesgo-rendimiento representada por la flecha en color azul. Las líneas punteadas en color rojo representan los promedios simples de los rendimientos (6.14\%) y del riesgo (15.97\%) de los diez fondos. Observando el comportamiento de los rendimientos, la Siefore que obtuvo el mayor rendimiento fue PensionISSSTE (7.41\%), con un nivel de bajo riesgo, incluso menor al promedio simpley muy cercano al fondo con menor riesgo que fue para Coppel (15.55\%). La segunda posición en generación de rendimientos fue para ProfuturoCNP (6.97\%), pero se expuso a un riesgo mayor (0.11\%) respecto a PensionISSSTE. En el tercer lugar en el pago de rendimientos se ubicó Sura (6.90\%) pero con un riesgo mayor (0.12\%) en comparación con ProfuturoCNP.

En contraste, observando el comportamiento del riesgo, las Siefores con la mayor exposición al riesgo de mercado fueron XXI Banorte (18.13\%) e Invercap (16.11\%), por lo tanto y de acuerdo con la relación riesgo-rendimiento deberían obtener los rendimientos más altos; sin embargo, XXI Banorte sólo genero rendimientos cercanos al promedio simple y en el caso de Invercap obtuvo los rendimientos más bajos de todos los fondos (4.76\%). Es importante mencionar que 
la diferencia entre la Sociedad que pagó el mayor rendimiento y el menor fue de $2.65 \%$. Respecto a la Siefore que obtuvo el mayor riesgo y el menor, la diferencia fue de $2.58 \%$, respectivamente.

\section{GRÁFICA 12. RIESCO PROMEDIO ANUALIZADO VS RENDIMIENTO PROMEDIO ANUALIZADO} PARA 10 SIEFORE DE LA SB3:2008-2019

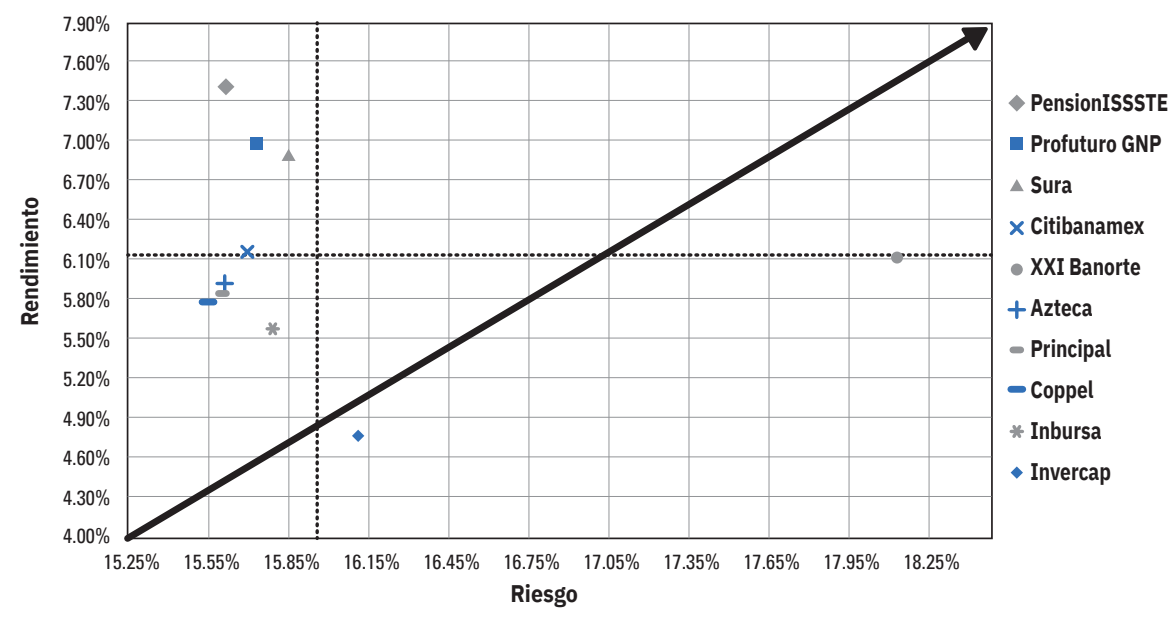

Fuente: elaboración propia

Por último, se realizó la comparación de los rendimientos estimados contra el IRN de la CONSAR, por lo que fue necesario ajustar la serie original de precios al horizonte de la Comisión de los últimos 84 meses, por lo tanto, las series de precios se analizaron del 28 de junio de 2013 al 28 de junio de 2019. Al respecto, se puede observar de manera general una alta coincidencia en el comportamiento de las curvas de ambos rendimientos (véase Gráfica 13). Los rendimientos estimados fueron superiores a los publicados por la Comisión (excepto para PensionISSSTEy Sura); sin embargo, se observó una alta coincidencia en aquella Sociedad con menor desempeño que fue ProfuturoGNP (fondo con mayores rendimientos para ambas metodologías) con un diferencial del $0.06 \%$ entre estas. Otra coincidencia importante se observó en la Sociedad que generó los rendimientos más bajos que fue Invercap (con un diferencial de 0.14\%). Las Siefores Inbursa y Citibanamex obtuvieron la mayor coincidencia en la comparativa ya que registraron los diferenciales más bajos (0.01\% y 0.04\%) en la comparativa. 
GRÁFICA 13. RENDIMIENTOS VS IRN DE LA CONSAR PARA

10 SIEFORE DE LA SB3 -ÚLTIMOS 84 MESES-

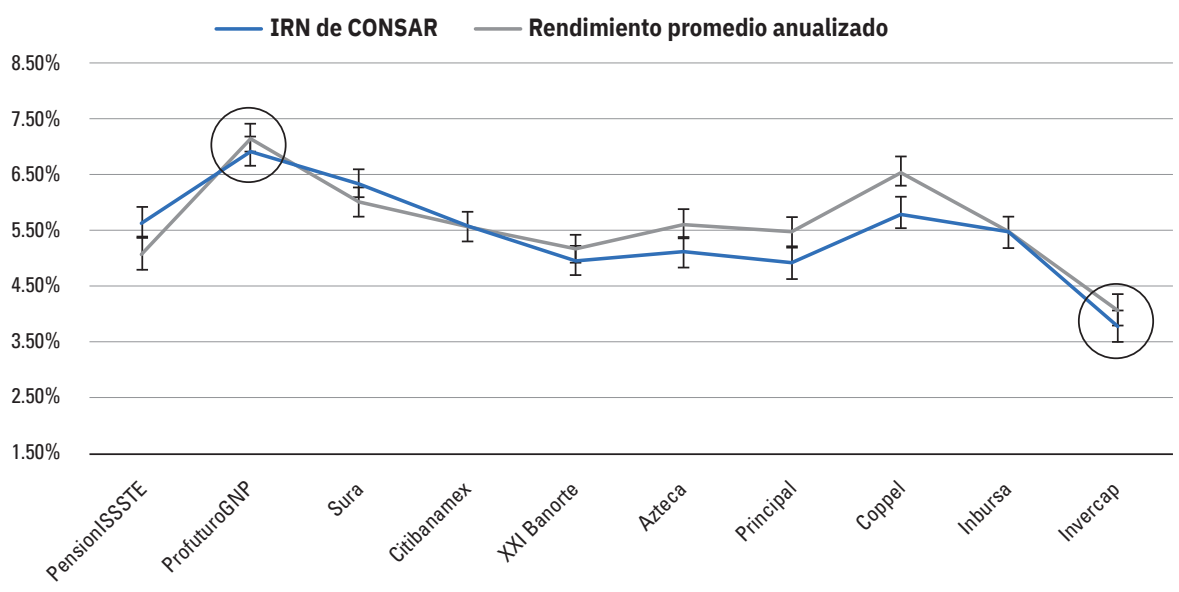

Fuente: elaboración propia

\section{Conclusiones}

Al término de la investigación se presentan los argumentos suficientes para aceptar la hipótesis planteada al inicio de ese trabajo que estableció que la relación riesgo-rendimiento no se cumple para las Siefores que conformaron la SB3 porque las inversiones con mayor riesgo, no generaron mayores rendimientos. El análisis econométrico permitió demostrar que las tres Siefores (PensionISSSTE, ProfuturoGNP y Sura) que pagaron los rendimientos más altos en el periodo de estudio, no expusieron sus inversiones al mayor riesgo de mercado. En contraste, la Sociedad que obtuvo el mayor riesgo de mercado fue XXI Banorte, por lo tanto, se esperaba que obtuviera los rendimientos más altos; sin embargo, generó bajos rendimientos y menores al promedio simple de todos los fondos. El caso más grave fue para Invercap, ya que generó los rendimientos más bajos de todos los fondos junto con una altísima exposición al riesgo.

Los casos anteriores (tanto del rendimiento y del riesgo) demostraron contundentemente que el esquema de inversión de multifondos de las Siefore, el cumplimiento del postulado financiero de a mayor riesgo mayor rendimiento no 
se cumple y para el caso de Invercap dicho postulado fue completamente nulo y contradice la teoría financiera. Lo anterior podría evidenciar una operación deficiente en el proceso de inversión por parte de dicha Siefore que al final podría afectar el monto de la pensión del trabajador en el largo plazo.

Por su parte, los rendimientos estimados comparados contra el IRN Neto de la CONSAR fueron altamente coincidentes (en la Siefore con la mayor y la menor generación de rendimientos) y superiores en ocho casos. Dicha comparativa no pretende funcionar como una guía para la selección de una Afore óptima para el ahorrador, únicamente se realizó con el propósito de encontrar coincidencia entre los rendimientos estimados y los del regulador. De esta manera al observar altas coincidencias con los rendimientos del regulador, fue posible realizar un análisis del riesgo-rendimiento más objetivo y presentar resultados con estimaciones probabilísticas más realistas sobre la operación de los ocho fondos estudiados.

Respecto al mecanismo de LSS que ofrece mayores rendimientos para los ahorradores (a cambio de un mayor nivel de riesgo) se demostró que en la práctica no fue financieramente viable, ya que los modelos econométricos demostraron que (por lo menos para la SB3) los fondos con mayor exposición al riesgo, no generaron mayores rendimientos. Además, la LSS contradice el proceso de inversión de las Afores que como se indicó se basa en el principio de responsabilidad fiduciaria ya que la Comisión le transfiere la responsabilidad al trabajador para elegir la Sociedad de inversión que prefiera; no obstante, dicha decisión debería ser tomada por el regulador tal como se planteó desde 1997 al asignar las Siefores de acuerdo al rango de edad del ahorrador. Bajo el contexto anterior, esta investigación considera que la LSS representa mayor riesgo para el trabajador (si es que decide cambiarse a una Siefore que ofrezca mayores rendimientos) ya que sería necesario que tuviera conocimientos financieros más allá de los básicos para poder evaluar la rentabilidad de su inversión y de la relación riesgo-rendimiento, la cual se establece (como hipótesis de este trabajo) que no se cumple para la SB3.

El problema de los rendimientos decrecientes de los fondos podría estar relacionado con la propia operación de los comités de inversión y de riesgos financieros de las Siefores y específicamente en sus estrategias de inversión para la compra-venta de instrumentos financieros, los rendimientos que esperan obtener y los riesgos que asumen. Lo anterior, representa una línea de investigación que podría analizarse si se cuenta con la información del proceso de inversión de una Siefore en particular. Finalmente, la última reforma a la LSAR para transitar 
de los multi fondos de las Siefores a fondos generacionales tiene el fin de generar mayores rendimientos, al respecto es importante realizar la medición futura de los mismos bajo este nuevo esquema, con el objetivo de conocer si efectivamente los rendimientos serán mayores en comparación al sistema anterior de Siefores.

\section{Bibliografía}

Banda, H.; González, L. y Gómez, D. (2014). "Una aproximación de la teoría de portafolio a las Siefores en México". Pensamiento y gestión 36, enero-junio, pp. 28-55. Universidad del Norte. Barranquilla, Colombia.

Banda, H. y Cómez, D. (2009). "Evaluación de un portafolio de inversión institucional: el caso de los fondos de pensiones en México". Innovaciones de Negocios, 6(2), pp. 303-323. Universidad Autónoma de Querétaro, Querétaro.

Blanco, F.; Ferrando, M. y Martínez, M. (2015). Teoría de la inversión. Ediciones Pirámide. Madrid.

Bonifant, L. y Cómez, D. (2010). Comparación de la tasa de reemplazo entre Afores. Universidad Autónoma de Querétaro, Querétaro.

Comisión Nacional del Sistema de Ahorro para el Retiro (2017). Boletín de Prensa $\mathrm{N}^{\circ} 07 / 2017$ sobre el cambio de Siefore.

Comisión Nacional del Sistema de Ahorro para el Retiro (2018). Diagnóstico del sistema de ahorro para el retiro en México: Funcionamiento, beneficios y retos.

Engle, R. y Patton, J. (2001). What a Good is a Volatility Model? Quantitative Finance,1(2), pp.237-245.

Ferruz, L. y Sarto, J. (2002). "Performance en la gestión de carteras en el contexto de la Teoría de la Utilidad en presencia de riesgo". Estudios de Economía Aplicada (20), pp. 81-110.

Gómez, M. (2016). Una propuesta de cambio al régimen de inversión 2015 de las Siefores. Tesis de Posgrado. Universidad Nacional Autónoma de México, CDMX.

Gujarati, Dy Porter, D. (2010). Econometría. Quinta Edición. Editorial, McGraw-Hill Interamericana.

Herrera, F. (2015). Riesgoy Rendimiento de la Siefore Básica 4 en México: Una Estrategia de Administración de Riesgos. Tesis de Posgrado. Universidad Nacional Autónoma de México, CDMX.

Jorion, P. (2004). Valor en riesgo. CDMX, Editorial Limusa. 
Martínez, M. y Venegas, F. (2014). "Análisis del riesgo de mercado de los fondos de pensión en México. Un enfoque con Modelos autorregresivos". Contaduría y Administración 59 (3), julio-septiembre, pp. 165-195. , CDMX, Universidad Nacional Autónoma de México.

Pokorny, M. (1987). "An Introduction to Econometrics”. Nueva York, Basil Blackwell.

Romero, G. (2016). Los sistemas de pensión en México: análisis del riesgo y rendimiento de los portafolios de las Siefores 2008-2012. Tesis de Posgrado. Instituto Politécnico Nacional, CDMX.

Santillán, R.; Martínez, M. y López, F. (2016). "Análisis econométrico del riesgo y rendimiento de las Siefores". Economía y Finanzas Nueva Época 11 (1), pp. 29-54.

Universidad Nacional Autónoma de México (2015). Análisis de Riesgo y Portafolios de Inversión. Facultad de Economía. Universidad Autónoma de México, CDMX. Véliz, G.; Cervantes, T.; y Carmona, E. (2012). "Análisis de riesgo vs. rendimiento de las acciones más volátiles que han cotizado en la BMV de 2003 a 2011". NovaRua 3(5), junio. Universidad Autónoma de Ciudad Juárez.

\section{Referencias Web}

Administración y Economía (2013). Rendimiento y Riesgo: conceptos básicos. Disponible en: http://admonyeconomia.blogspot.com/2012/05/rendimiento-y-riesgo-conceptos-basicos.html [Consultado enero, 2018].

Banco de México (2005). Definiciones básicas de riesgos. Disponible en: https://www. banxico.org.mx/AplBusquedasBM2/bgenwww_es.jsp [Consultado marzo, 2018].

Bollerslev, T. (1986). "Ceneralized autoregressive conditional heteroskedasticity". Journal of Econometrics 31 (3) abril, pp. 307-327. Disponible en: https://econpapers. repec.org/article/eeeeconom/v_3a31_3ay_3a1986_3ai_3a3_3ap_3a307-327.htm [Consultado enero, 2018].

Catalán, H. (2011). Especificación y estimación de los modelos ARCH. Disponible en: https://www.cepal.org/sites/default/files/events/files/cc_11.2011.horario.catalan. modelosarch.esp_.pdf [Consultado febrero, 2018].

Comisión Nacional del Sistema de Ahorro para el Retiro (2017). Radiografía del sistema de pensiones a 20 años de su creación. Disponible en: https://www.gob.

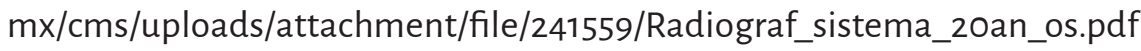
[Consultado febrero, 2018].

(2018a). Panorama Ceneral del SAR. Disponible en: https://www.gob.mx/consar/ documentos/panorama-general-del-sar-56798?idiom=es 
[Consultado marzo, 2018].

(2019) Modificación del Régimen de Inversión para transitar de un esquema de multifondos a un esquema de fondos generacionales. Disponible en: https://www. gob.mx/consar/prensa/se-modifica-el-regimen-de-inversion-para-transitar-de-un-esquema-de-multifondos-a-un-esquema-de-fondos-generacionales-202764?idiom=es [Consultado julio, 2018].

Engle, R. (2004). "Riesgo y volatilidad: modelos econométricos y practica financiera". Asturiana de Economía 31, New York University. Disponible en: http://www.revistaasturianadeeconomia.org/raepdf/31/ENCLE.pdf [Consultado marzo, 2018].

e-SAR (2018). Información sobre el SAR en México. Disponible en: https://www.e-sar. com.mx/PortalEsar/public/sieforelnfo.do [Consultado julio, 2018] .

Markowitz, H. (1952). "Portfolio Selection”. The Journal of Finance 7(1), pp. 77-91.Disponible en: https://www.jstor.org/stable/2975974?seq=1\#page_scan_tab_contents [Consultado marzo,2018]. 